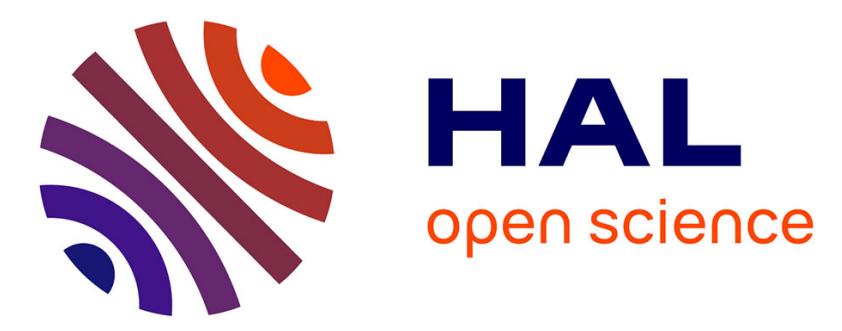

\title{
ELASTIC- Enabling Massive-Antenna for Joint Spectrum Sensing and Sharing: How Many Antennas Do We Need?
}

Hussein Kobeissi, Youssef Nasser, Oussama Bazzi, Amor Nafkha, Yves Louet

\section{- To cite this version:}

Hussein Kobeissi, Youssef Nasser, Oussama Bazzi, Amor Nafkha, Yves Louet. ELASTIC- Enabling Massive-Antenna for Joint Spectrum Sensing and Sharing: How Many Antennas Do We Need?. IEEE Transactions on Cognitive Communications and Networking, 2019, 5 (2), pp.267-280. 10.1109/TCCN.2019.2901847 . hal-02151864

\section{HAL Id: hal-02151864 https://hal.science/hal-02151864}

Submitted on 8 Jul 2019

HAL is a multi-disciplinary open access archive for the deposit and dissemination of scientific research documents, whether they are published or not. The documents may come from teaching and research institutions in France or abroad, or from public or private research centers.
L'archive ouverte pluridisciplinaire HAL, est destinée au dépôt et à la diffusion de documents scientifiques de niveau recherche, publiés ou non, émanant des établissements d'enseignement et de recherche français ou étrangers, des laboratoires publics ou privés. 


\title{
ELASTIC- Enabling Massive-Antenna for Joint Spectrum Sensing and Sharing: How Many Antennas Do We Need?
}

\author{
Hussein Kobeissi*, Youssef Nasser*, Oussama Bazzi, Amor Nafkha, and Yves Louët
}

\begin{abstract}
Massive Antenna and Cognitive Radio (CR) technologies have attracted many research interests due to the additional resources offered in striving against the spectrum crisis. In this paper, we propose a general framework to EnlabLe mASsive anTenna exploItation for spectrum sensing and sharing in CR (ELASTIC). Using random matrix theory and moment matching method, we derived a simple approximation of the distributions of three eigen-value based detectors namely the Largest Eigenvalue (LE), the Scaled LE (SLE), and Standard Condition Number (SCN). This has led to a simple analytical formulation and optimization of the number of antennas and the number of samples to reach a target performance. To exploit the large number of antennas, we proposed two sensing scenarios. The first is based on full antenna exploitation and guarantees an optimal performance despite the transmission conditions. The second is based on partial antenna exploitation, which determines the exact number of antennas and samples required to reach the target performance. We have shown that the framework offers an additional degree of freedom in the selection of the optimal system parameters, namely the number of antennas, while the remaining antennas are exploited for sharing in other dimensions of the spectrum hypercube.
\end{abstract}

Index Terms-Cognitive radio, MIMO systems, Eigenvalue based detector, Spectrum sensing, Wishart matrix.

\section{INTRODUCTION}

$5^{\mathrm{G}}$ $\mathrm{G}$, the fifth generation of mobile networks, is expected to accommodate new demands and high data-rates that are growing at an unprecedented pace requiring a large amount of radio frequency resources. To fulfill these requirements, the Cognitive Radio (CR) technology has been introduced to offer a more efficient use of spectrum and thus reduce its scarcity [1]. A CR device can sense an unused channel and adjust its transceiver parameters accordingly.

In CR networks, Spectrum Sensing (SS) is the task of obtaining awareness about the spectrum usage. It concerns two scenarios of detection: (i) detecting the absence of the Primary User (PU) in a licensed spectrum and (ii) detecting the presence of the PU to avoid interference. Hence, SS plays a major role in the performance of the CR technology for both

Hussein kobeissi was with the Signal, Communication, and Embedded Electronics (SCEE-IETR) research group at CentraleSuplec, Rennes, France and also with the Doctoral School of Science and Technology, Lebanese University, Beirut, Lebanon. He is with the Computer and Communication Engineering Department of the International University of Beirut (BIU), Beirut, Lebanon (e-mail: hussein.kobeissi@liu.edu.lb).

Youssef Nasser is with ECE Department, American University of Beirut, Bliss Street, Beirut, Lebanon (e-mail: youssef.nasser@ieee.org)

Oussama Bazzi is with Department of Physics and Electronics, Faculty of Science 1, Lebanese University, Hadath, Beirut, Lebanon (email: obazzi@ul.edu.lb)

Yves Louët and Amor Nafkha are with SCEE/IETR, CentraleSupélec of Rennes, Rennes, France (e-mail: Yves.Louet, Amor.Nafkha@centralesupelec.fr).

(*): both are co-first authors the PU and Secondary User (SU). In this regard, several SS algorithms have been proposed [2]. These techniques include energy detector, matched filter detector, cyclostationary feature detector, etc [3]. SS techniques with superior performance and robustness were also designed using the eigenvalues of the received signal's covariance matrix. These detectors, classified under the name eigenvalue based detector (EBD), rely on the use of random matrix theory (RMT) and different eigenvalue properties of the sample covariance matrix in decision making. The key advantage of the EBD lies in the fact that it can reach a high sensing performance without necessarily requiring knowledge about the primary signal and the noise power. EBD techniques include, but are not limited to, the largest eigenvalue (LE) detector [4]-[7], the scaled largest eigenvalue (SLE) detector [7]-[15], and the standard condition number detector (SCN) [4], [6], [16]-[20].

In the context of massive antenna deployment (up to few hundred in the literature), it is very likely for CRs to exploit the advantages offered by the multiple-input multiple-output (MIMO) technologies to improve secondary communications [21]-[26]. For instance, massive antenna technology allows the secondary users exploiting the angle-of-arrival dimension in the transmission hyperspace using beamforming [27]-[30]. As such, CR could be combined with massive MIMO through the additional degree of freedom offered by the large number of antennas to identify the unused channels while achieving a significant increase in the performance of the SS detector. It might be also enough to use a fewer number of antennas for the sensing process while the rest could be used for other purposes. Hence, two scenarios could be considered:

1. Full Antenna Exploitation (FAE) scenario: Therein, the CR module may use all of its antennas to detect primary users and hence it reduces the number of samples required to perform spectrum sensing. Thus, the sensing time overhead is reduced.

2. Partial antenna exploitation (PAE) scenario: the CR module may set a certain number of antennas to perform primary users detection and use the rest of the antennas for other purposes (mainly transmission). It may also dynamically change the number of antennas $K$ and/or the number of samples $N$ according to predefined performance, and use the remaining antennas for other purposes.

Accordingly, the calculation of the minimum number of antennas and/or the minimum number of samples required to reach a given target performance is very important. However, these values are directly related to the statistics of the spectrum sensing approach and its corresponding performance metrics and mechanisms. 
In this paper, we mainly consider the framework to EnlabLe mASsive anTenna exploItation for spectrum sensing and sharing CR (ELASTIC). We firstly introduce the LE, SLE and SCN detectors, and we propose new approximations for their distribution based on the generalized extreme value (GEV) probability density function (PDF). These detectors are then considered and studied in the two antenna exploitation scenarios, i.e. FAE and PAE. The main contributions of this paper can be summarized as follows:

- Proposition of a new approximation for the cumulative distribution function (CDF) and the PDF of the LE detector.The proposed approximation provides a simple formulation of the proposed analytical framework and allows an optimization of the number of antennas as stated by ELASTIC. To the best of the authors knowledge, this approximation has not been proposed in literature including our previous works.

- Extension of our previous work in [14], [19], [20] to provide key design metrics for the operators to optimize the CR network. Indeed, in our previous works, we have derived new approximations of the PDF EBD detectors. These approximations led to simple formulations that could be exploited in the design of a system with multiple antennas. In ELASTIC, we exploit these formulations to set and derive key design metrics depending on the required system performance given in terms of probability of false alarm and detection. In other words, this work proposes a simple analytical tool to tune these metrics (such as detection threshold) in terms of the network transmission parameters (as the number of antennas ${ }^{1}$.

- Derivation of the optimal decision thresholds for the three detectors in both FAE and PAE scenarios. The latter is given in terms of probability of false alarm $P_{f a}$ and probability of detection $P_{d}$.

- Derivations of the minimum requirements in number of antennas $K$ and number of samples $N$ that achieve a target detection performance.

Note 1: This work shows that a reduced number of antennas is needed to sense a channel. Accordingly, in a massive antenna environment, a large number of channels can be sensed simultaneously.

Note 2: It is worthy that the exact distributions of the three detectors have been derived in literature. However, these distributions are too complicated to be used in the analytical optimization of the system metrics. For instance, a MarchenkoPastur (MP) law is used for the SCN distribution [4] but its expression is hard to be analytically exploited.

To the best of the authors knowledge, this work is a first of its kind in literature and will definitely open new research pathways to exploit the analytical derivations provided in this paper to other applications such as spectrum sharing, cognitive user capacity analysis, mmWave base station massive sensing capabilities in vehicular networks [31], etc.

\footnotetext{
${ }^{1}$ The paper does not reproduce our previous work but provides a reminder on the main derivations that have been stated to assure a smooth readability of the current work. These derivations are then extended to optimize the Massive-MIMO CR parameters.
}

The rest of this paper is organized as follows. In section II, we present the main rationale behind ELASTIC framework and give the benefits of these approaches. Section III introduces the system framework, system model and defines the main parameters for EBD. In Section IV, the largest eigenvalue detector is firstly introduced and then followed by the proposed approximation of its PDF in different scenarios. In Sections V and VI, the SLE and and SCN detectors are introduced and then followed by the proposed PDF distributions respectively. Full and partial antenna exploitation scenarios are analytically considered in section VII. Threshold derivation and optimization are considered along with optimal system requirements in Section VIII. Results are validated and discussed in section IX while the conclusion is drawn in section $\mathrm{X}$.

Notations: Vectors and Matrices are represented, respectively, by lower and upper case boldface. The symbols $|\cdot|$ and $\operatorname{tr}($.$) indicate, respectively, the determinant and trace of$ a matrix while $(.)^{1 / 2},(.)^{T}$, and $(.)^{\dagger}$ are the square root, transpose, and Hermitian symbols respectively. $\boldsymbol{I}_{n}$ is the $n \times n$ identity matrix and $\mathbf{1}_{K N}$ is a $K \times N$ ones matrix. Symbol $\sim$ stands for "distributed as" and $E[$.$] stands for the expected$ value.

\section{The Rationale Behind ELASTIC}

As stated earlier, two main scenarios could be considered: the FAE and the PAE. While the full antenna exploitation scenario is using all of the antennas at the same time for spectrum sensing, the partial antenna exploitation scenario could be decomposed into two different approaches: (i) exploit a fixed number of antennas for SS and thus use the rest for other purposes, it will be labeled fixed PAE (FPAE) and (ii) a dynamic approach in which the CR does not set a predefined number of antennas but it dynamically allocates a certain number of antennas for SS according to certain constraints, labeled dynamic PAE (DPAE).

The division into approaches could be justified by the simple fact that a trade-off between spectrum sensing performance and exploitation of available antennas for other targets could exist. This depends on the different metrics and performance objectives. In this work, the following approaches could be considered:

- Sensing multi-channels simultaneously [32], [33]: Within the framework of ELASTIC, a new approach is considered: the antennas are clustered so that each set is used to sense certain channel and/or certain direction. Hence, several spectrum holes could be found at the same time.

- Sense and transmit simultaneously: Contrarily to the conventional sensing techniques [34], simultaneous sensing and transmission could be considered as a full duplex approach in which two RF chains are required at the CR receiver [35]. Likewise, the $\mathrm{CR}$ could maintain a set of antennas for SS and the others for the transmission in a full duplex scenario.

- Green radio: ELASTIC framework offers an energy efficiency perspective in which a limited number of antennas are switched-on while the others are in sleep mode [36].

- Reduce sensing time: The FAE scenario may not be the optimal scheme to reduce the sensing time since PAE 
could offer the same performance with less antennas. Hence, an optimal trade-off between the number of antennas and the required number of samples to make a decision may decrease the total sensing time.

- Increase system throughput: In general, using some or all of these methods would increase the total system throughput as the system will be able to transmit data over the remaining time/antennas.

Consequently, the use of a fixed number or dynamic number of antennas for spectrum sensing is extremely important for $\mathrm{CR}$ with massive antenna technology. In both cases, the CR technology has to optimize the number of antenna $K$ and samples $N$ to perform primary users detection so that to maintain a target performance for any transmission conditions (given for e.g. in terms of Signal-to-Noise Ratio SNR). In both cases, the remaining number of antennas will be used for other transmission purposes.

\section{ELASTIC FRAMEWORK}

\section{A. System Model}

Consider a multiple-antenna $\mathrm{CR}$ system, say $K$ antennas, aiming to detect the presence/absence of a single PU during a sensing period equivalent to $N T_{s}$ where $T_{s}$ is the sampling period. For this detection problem, there are two hypotheses: the null hypothesis $\mathcal{H}_{0}$ corresponds to the absence of PU transmission (i.e. spectrum hole); and the alternative hypothesis $\mathcal{H}_{1}$ corresponds to the presence of the PU transmission (i.e. spectrum being used). The received vector, at instant $n T_{s}$, under both hypotheses is given by:

$$
\begin{aligned}
& \mathcal{H}_{0}: \boldsymbol{y}(n)=\boldsymbol{b}(n), \\
& \mathcal{H}_{1}: \boldsymbol{y}(n)=\boldsymbol{h}(n) s(n)+\boldsymbol{b}(n),
\end{aligned}
$$

where $\boldsymbol{y}(n)=\left[y_{1}(n), \cdots, y_{K}(n)\right]^{T}$ is the observed $K \times 1$ complex samples received from different antennas at instant $n$. $\boldsymbol{b}(n)$ is a $K \times 1$ complex circular white Gaussian noise with zero mean and variance $\sigma_{b}^{2} \cdot \boldsymbol{h}(n)$ is a $K \times 1$ vector that represents the channels' coefficients between the PU and each antenna at the $\mathrm{CR}$ receiver and $s(n)$ stands for the primary signal sample at instant $n T_{s}$, having a Gaussian distribution with zero mean and variance $\sigma_{s}^{2}$. In this paper, under $\mathrm{H}_{1}$, the model represents a single PU transmitted over an independent and identically distributed (i.i.d.) channel coefficients with zero mean and variance $\sigma_{h}^{2}$ or equivalently the PU signal is sampled subject to uncorrelated (fast) Rayleigh fading. This assumption is in line with the literature works such [18] [24]. Moreover, the channel coefficients $h(n)$ are assumed to be i.i.d. at each time instant $n$. After collecting $N$ samples from each antenna, the received signal matrix $\boldsymbol{Y}$ is written as:

$$
\boldsymbol{Y}=\left(\begin{array}{cccc}
y_{1}(1) & y_{1}(2) & \cdots & y_{1}(N) \\
y_{2}(1) & y_{2}(2) & \cdots & y_{2}(N) \\
\vdots & \vdots & \ddots & \vdots \\
y_{K}(1) & y_{K}(2) & \cdots & y_{K}(N)
\end{array}\right)
$$

Without loss of generality, we suppose that $K \leq N$ and we define the received sample covariance matrix as $\boldsymbol{W}=\boldsymbol{Y} \boldsymbol{Y}^{\dagger}$.
1) $\mathcal{H}_{0}$ hypothesis: Under $\mathcal{H}_{0}$ hypothesis, the entries of the matrix $\boldsymbol{Y}$ are complex Gaussian with zero mean and variance $\sigma_{b}^{2}$. Therefore, the sample covariance matrix, $\boldsymbol{W}$, is a $K \times K$ central uncorrelated complex Wishart matrix with $N$ degrees of freedom (DoF) and with statistical covariance matrix:

$$
\boldsymbol{\Sigma}=\sigma_{b}^{2} \mathbf{I}_{K}
$$

and is denoted by $\mathbf{W} \sim \mathcal{C W}_{K}\left(N, \sigma_{b}^{2} \mathbf{I}_{K}\right)$.

2) $\mathcal{H}_{1}$ hypothesis: Under $\mathcal{H}_{1}$, we assume the presence of a single PU. Consequently, $\mathbf{W}$ follows a central semi-correlated complex Wishart distribution, denoted by $\mathbf{W} \sim \mathcal{C W}_{K}(N, \boldsymbol{\Sigma})$, with $N$ DoF and statistical covariance matrix $\boldsymbol{\Sigma}$ given by [37]:

$$
\boldsymbol{\Sigma}=\sigma_{s}^{2} \boldsymbol{h}^{\dagger}+\sigma_{b}^{2} \mathbf{I}_{K}
$$

The statistical covariance matrix $\boldsymbol{\Sigma}$ is a rank 1 perturbation of the identity matrix, then it belongs to the class of spiked population model first introduced by [38]. Since the rank of $\sigma_{s}^{2} \boldsymbol{h} \boldsymbol{h}^{\dagger}$ is 1 , then all but one of the eigenvalues of $\boldsymbol{\Sigma}$ are still $\sigma_{b}^{2}$. Denoting the average SNR by:

$$
\rho=\frac{\sigma_{s}^{2} \sigma_{h}^{2}}{\sigma_{b}^{2}},
$$

and by using the property that the trace of a matrix equals the sum of its eigenvalues, the eigenvalues of $\boldsymbol{\Sigma}$ are given by:

$$
\boldsymbol{\sigma}=\sigma_{b}^{2}\left[K \rho+1, \mathbf{1}_{1, K-1}\right]
$$

\section{B. Eigenvalue Based Detector}

EBD could be divided into two classes: detectors that require the knowledge of noise variance and detectors that do not require this knowledge. LE detector is the optimal detector when noise variance is perfectly known while SLE detector is the optimal under the generalized likelihood ratio (GLR) criterion when noise variance is unknown [7], [10]. Other EBD techniques such as the SCN detector provide good performance while they don't require channel knowledge or primary user information. For a given decision threshold $\hat{\lambda}_{E B D}$, the general EBD algorithm is given by:

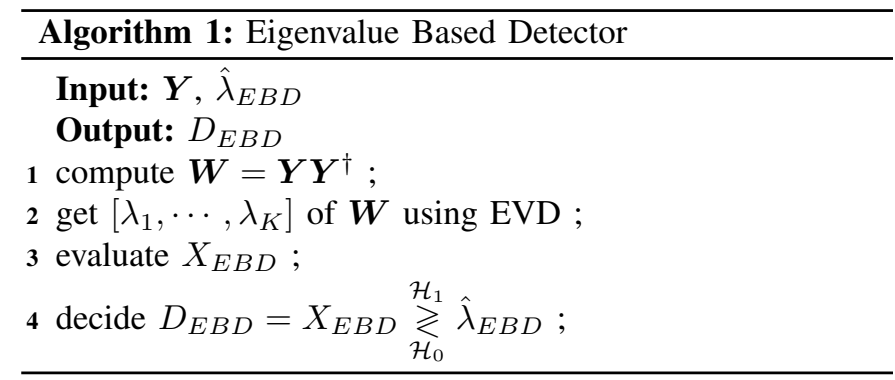

where $\left[\lambda_{1}, \cdots, \lambda_{K}\right]$ is the vector of eigenvalues of the covariance matrix $\boldsymbol{W}, X_{E B D}$ is the EBD metric used for detection and $D_{E B D}$ is its decision.

\section{Performance Probabilities}

The performance of any spectrum sensing technique is usually expressed in terms of its false-alarm probability and its missed-detection probability, both known as the error 
probabilities. The probability of false-alarm probability $\left(P_{f a}\right)$ and the probability of missed-detection $\left(P_{m d}\right)$ are defined as follows:

$$
\begin{gathered}
P_{f a}=\operatorname{Pr}\left(X \geq \alpha / \mathcal{H}_{0}\right) \\
P_{m d}=\operatorname{Pr}\left(X<\alpha / \mathcal{H}_{1}\right)
\end{gathered}
$$

where $X$ denotes the statistical EBD metric.

\section{Operation Conditions of the EBD Derivations}

In this paper, two different conditions will be distinguished. 1) Asymptotic Condition $(A C)$ : The $\mathrm{AC}$ is given by:

$$
(K, N) \rightarrow \infty \text { with } K / N \rightarrow r \in(0,1)
$$

2) Critical Condition $(C C)$ : The critical condition is related with the SNR requirement by:

$$
\rho>\rho_{c}=\frac{1}{\sqrt{K N}},
$$

\section{LARGEST EIGENVALUE DETECTOR}

The LE statistical metric is defined as the ratio between the largest eigenvalue of the received covariance matrix and the noise power. It can be expressed as follows:

$$
X_{L E}=\frac{\lambda_{1}}{\sigma_{b}^{2}}
$$

Its exact distribution is derived in literature in the form of matrix determinant (see, for instance, [39], [40]).

\section{A. LE Detector in Asymptotic Regime}

The asymptotic regime is defined in this paper when (10) is verified. Asymptotically, $X_{L E}$ metric follows a Tracy-Widom (TW) distribution for central uncorrelated Wishart matrices (i.e. $\mathcal{H}_{0}$ ) and a Gaussian distribution for sample covariance matrices of spiked population model (i.e. $\mathcal{H}_{1}$ ) [41]. However, for a fixed $K$ and as $N \rightarrow+\infty$ then the metric $X_{L E}$ converges to a Gaussian distribution [42].

1) Null Hypothesis Case: By considering the AC in (10), $X_{L E}$, properly centered and scaled, follows asymptotically a TW distribution of order 2 (TW2) as follows:

$$
X_{L E}^{\prime}=\frac{X_{L E}-a_{1}(K, N)}{b_{1}(K, N)} \sim T W 2,
$$

where $a_{1}(K, N)$ and $b_{1}(K, N)$ are respectively the centering and scaling coefficients defined by:

$$
\begin{aligned}
& a_{1}(K, N)=(\sqrt{K}+\sqrt{N})^{2}, \\
& b_{1}(K, N)=(\sqrt{K}+\sqrt{N})\left(K^{-1 / 2}+N^{-1 / 2}\right)^{\frac{1}{3}} .
\end{aligned}
$$

2) $\mathcal{H}_{1}$ Hypothesis Case: Under the asymptotic condition in (10) and the critical condition in (11), it has been shown that $X_{L E}$ follows a normal distribution such that [43]:

$$
P\left(\frac{X_{L E}-a_{2}(K, N, \sigma)}{\sqrt{b_{2}(K, N, \sigma)}} \leq x\right)=\frac{1}{\sqrt{2 \pi}} \int_{-\infty}^{x} e^{-\frac{u^{2}}{2}} d u
$$

with

$$
\begin{aligned}
& a_{2}(K, N, \sigma)=\sigma_{1}\left(N+\frac{K}{\sigma_{1}-1}\right) \\
& b_{2}(K, N, \sigma)=\sigma_{1}^{2}\left(N-\frac{K}{\left(\sigma_{1}-1\right)^{2}}\right)
\end{aligned}
$$

where $\sigma_{1}$ is the largest eigenvalue of (7). Definitely, if $\rho<\rho_{c}$, the null hypothesis case stands in which $X_{L E}$, under (10), follows the TW2 distribution [43]. Accordingly, the PU signal has no effect on the eigenvalues and could not be detected.

\section{B. Approximating $X_{L E}$ distribution under $\mathcal{H}_{0}$}

It has been shown that, for a fixed $K$ and as $N \rightarrow \infty$, $X_{L E}$ follows a normal distribution [42]. However, from a FAE perspective, i.e. both $K$ and $N$ are large and $\mathrm{AC}$ is satisfied, $X_{L E}$ follows the TW2 distribution rather than the normal distribution. TW2 distribution requires, from one side, the calculation of complex derivations that include special functions and, from the other side, these derivations depend on many transmission parameters. Hence, these derivations are not analytically tractable for the CR system metrics optimization (for instance optimal $K$ and $N$ ). Accordingly, two alternatives may be used to avoid this expensive calculation: (i) use a lookup table (LUT) approach or (ii) approximate its PDF by simpler expression. The LUT is interesting as it provides an off-line calculation of the system metrics however it might be too expensive in case of dynamic antenna exploitation. In this paper, we propose a new approximation for $X_{L E}$ distribution by using the GEV distribution and the moment matching method. Our objective is to find a simpler form that could be analytically used in the optimization problem of both FAE and PAE. Now, considering the asymptotic condition in (10), the mean, variance and skewness of the $X_{L E}$ are given by the following Corollary:

Corollary 1. Let $\mathbf{W} \sim \mathcal{C W}_{K}\left(N, \sigma_{b}^{2} \mathbf{I}_{K}\right)$ be a central uncorrelated complex Wishart matrix with $N$ DoF and correlation matrix $\sigma_{b}^{2} \mathbf{I}_{K}$. If $K$ and $N$ obey (10), then the mean, variance and skewness of $X_{L E}$ associated to $\mathbf{W}$ are given, respectively, by:

$$
\begin{aligned}
& \mu_{X_{L E}}=b_{1}(K, N) \mu_{T W 2}+a_{1}(K, N), \\
& \sigma_{X_{L E}}^{2}=b_{1}^{2}(K, N) \sigma_{T W 2}^{2}, \\
& \mathcal{S}_{X_{L E}}=\mathcal{S}_{T W 2},
\end{aligned}
$$

with $\mu_{T W 2}=-1.7710868074, \sigma_{T W 2}^{2}=0.8131947928$ and $\mathcal{S}_{T W 2}=0.2240842036$ are, respectively, the mean, variance and skewness of the TW2 distribution [44].

Proof: This result comes directly from (13).

Fortunately, the mean, variance and skewness expressions of $X_{L E}$ have simple forms when asymptotic condition in (10) is achieved. In this regard, the following proposition is useful. 
Proposition 1. Let $N$ and $K$ obey (10), i.e. under $A C$, then the CDF and PDF of the LE under null hypothesis can be accurately approximated respectively by:

$$
\begin{aligned}
F(x ; \theta, \beta, \xi) & =e^{-\left(1+\left(\frac{x-\theta}{\beta}\right) \xi\right)^{-1 / \xi}} \\
f(x ; \theta, \beta, \xi) & =\frac{1}{\beta}\left(1+\left(\frac{x-\theta}{\beta}\right) \xi\right)^{\frac{-1}{\xi}-1} e^{-\left(1+\left(\frac{x-\theta}{\beta}\right) \xi\right)^{-1 / \xi}}
\end{aligned}
$$

where $\xi, \beta$, and $\theta$ are respectively the shape, scale and location parameters of the GEV distribution and are given by:

$$
\begin{aligned}
\xi & =-0.06393 \mathcal{S}_{X_{L E}}^{2}+0.3173 \mathcal{S}_{X_{L E}}-0.2771 \\
\beta & =\sqrt{\frac{\sigma_{X_{L E}}^{2} \xi^{2}}{g_{2}-g_{1}^{2}}} \\
\theta & =\mu_{X_{L E}}-\frac{\left(g_{1}-1\right) \beta}{\xi}
\end{aligned}
$$

and the mean, the variance and the skewness of $X_{L E}$ are given by Corollary 1.

Proof: The result can be found using Lemma 5 of [45].

It follows from Proposition 1 that TW2 distribution, itself, could be approximated using GEV distribution by considering the mean, the variance and the skewness of TW2 mentioned in Corollary 1. Moreover, it will be shown through simulation results that this approximation is valid even if $K$ and $N$ are not asymptotically large.

\section{Approximating $X_{L E}$ distribution under $\mathcal{H}_{1}$}

For $\mathcal{H}_{1}$ hypothesis, $X_{L E}$ follows the Gaussian distribution [42]; it is indeed simple to exploited and any approximation in this case is useless.

\section{SLE DETECTOR}

The SLE detector is a blind detector that does not require information about the noise power. The statistical metric is defined as the ratio of the largest eigenvalue to the normalized trace of the received covariance matrix, and it is given by:

$$
X_{S L E}=\frac{\lambda_{1}}{T_{n}}=\frac{\lambda_{1}}{\frac{1}{K} \sum_{i=1}^{K} \lambda_{i}}
$$

In the literature, the results on the statistics of the SLE are relatively limited. They are based on tools from RMT [8], [10], [46] and Mellin transform [11], [13], [46]. $X_{S L E}$ was considered, asymptotically, to follow the same distribution as the $X_{L E}$ (i.e. TW distribution) [10]. However, as a nonnegligible error still exists in this approximation, a new form was provided based on the TW distribution and its second derivative in [8].

1) Null Hypothesis Case: Under $\mathcal{H}_{0}$, both the $X_{L E}$ and the normalized trace of the matrix $\mathbf{W}$ follow the Gaussian distribution as $N \rightarrow+\infty$ which is realistic in practical spectrum sensing scenarios. Accordingly, we have shown that the SLE detector can be formulated using standard Gaussian function as follows [14], [15]:
Theorem 1. Let $X_{S L E}$ be the statistical metric associated to $\mathbf{W} \sim \mathcal{C W}_{K}\left(N, \sigma_{b}^{2} \mathbf{I}_{K}\right)$. Then, for a fixed $K$ and as $N \rightarrow+\infty$, the $C D F$ and the PDF of $X_{S L E}$ are, respectively, given by:

$$
\begin{gathered}
F(x)=\Phi\left(\frac{x \mu_{T_{n}}-\mu_{\lambda_{1}}}{\sqrt{\sigma^{2}{ }_{\lambda_{1}}-2 x c+x^{2} \sigma^{2} T_{n}}}\right) \\
f(x)=\frac{\mu_{T_{n}} \sigma^{2}{ }_{\lambda_{1}}-c \mu_{\lambda_{1}}+\left(\mu_{\lambda_{1}} \sigma^{2} T_{n}-c \mu_{T_{n}}\right) x}{\left(\sigma^{2} \lambda_{1}-2 x c+x^{2} \sigma^{2} T_{n}\right)^{\frac{3}{2}}} \\
\times \phi\left(\frac{x \mu_{T_{n}}-\mu_{\lambda_{1}}}{\sqrt{\sigma^{2} \lambda_{1}-2 x c+x^{2} \sigma^{2} T_{n}}}\right)
\end{gathered}
$$

with

$$
\Phi(v)=\int_{-\infty}^{v} \phi(u) d u \quad \text { and } \quad \phi(u)=\frac{1}{\sqrt{2 \pi}} e^{-\frac{u^{2}}{2}}
$$

where $\mu_{\lambda_{1}}, \mu_{T_{n}}$ and $\sigma_{\lambda_{1}}^{2}, \sigma^{2} T_{n}$ are, respectively, the mean and the variance of $\lambda_{1}$ given by (19), (31) and (20), and $T_{n}$ given by (32). The parameter $c$ is given by $c=\sigma_{\lambda_{1}} \sigma_{T_{n}} \gamma$ where $\gamma$ is the correlation coefficient between $\lambda_{1}$ and $T_{n}$ provided by (33).

$$
\begin{aligned}
& \mu_{T_{n}}=N, \\
& \sigma^{2} T_{n}=N / K, \\
& \sigma=\frac{\sigma_{T_{n}}}{\sigma_{\lambda_{1}}} \cdot \frac{\theta \mu_{X_{S L E}}-\mu_{\lambda_{1}}}{\theta+\mu_{T_{n}}} .
\end{aligned}
$$

and $\mu_{X_{S L E}}$ is the mean of $X_{S L E}$ provided by (34) and $\theta$ is given by (35).

$$
\begin{aligned}
& \mu_{X_{S L E}}=\frac{\mu_{\lambda_{1}}}{\mu_{T_{n}}} \\
& \theta=1.01 \mu_{T_{n}}-0.2713 \sigma_{T_{n}}
\end{aligned}
$$

Proof: The CDF is given by (28) and the PDF is its derivative in (29) [47]. The reader can refer to [14], [15] for more details.

2) $\mathcal{H}_{1}$ Hypothesis Case: Under $\mathcal{H}_{1}$, the normalized trace follows the Gaussian distribution as $N \rightarrow+\infty$ whereas the LE follows the Gaussian distribution as $(K, N) \rightarrow+\infty$ with $K / N \rightarrow r \in(0,1)$ and $\rho>\rho_{c}=1 / \sqrt{K N}$. Accordingly, the distribution of the SLE is given by Theorem 2 [15].

Theorem 2. Let $X_{S L E}$ be the statistical metric associated to $\mathbf{W} \sim \mathcal{C W}_{K}(N, \boldsymbol{\Sigma})$. Then, as $(K, N) \rightarrow+\infty$ with $K / N \rightarrow r \in(0,1)$ and $\rho>\rho_{c}=1 / \sqrt{K N}$, the $C D F$ and PDF of $X_{S L E}$ are, respectively, given by (28) and (29). Moreover, $\mu_{\lambda_{1}}, \mu_{T_{n}}$ and $\sigma_{\lambda_{1}}^{2}, \sigma^{2} T_{n}$ are, respectively, the mean and the variance of $\lambda_{1}$ and $T_{n}$ given by (36), (38) and (37), (39) respectively where $\sigma_{1}$ is the largest eigenvalue of (7). The parameter $c$ is defined by $c=\sigma_{\lambda_{1}} \sigma_{T_{n}} \gamma$ where $\gamma$ is the correlation coefficient between $\lambda_{1}$ and $T_{n}$ given by (40).

$$
\begin{aligned}
& \mu_{\lambda_{1}}=\sigma_{1}\left(N+\frac{K}{\sigma_{1}-1}\right), \\
& \sigma^{2}{ }_{\lambda_{1}}=\sigma_{1}^{2}\left(N-\frac{K}{\left(\sigma_{1}-1\right)^{2}}\right), \\
& \mu_{T_{n}}=\frac{N}{K}\left(\sigma_{1}+K-1\right), \\
& \sigma^{2} T_{n}=\frac{N}{K^{2}}\left(\sigma_{1}^{2}+K-1\right), \\
& \gamma=\frac{\sigma_{T_{n}}}{\sigma_{\lambda_{1}}} \cdot \frac{\theta\left(\mu_{X}+\epsilon\right)-\mu_{\lambda_{1}}}{\theta+\mu_{T_{n}}}
\end{aligned}
$$


and $\mu_{X_{S L E}}$ is the mean of $X_{S L E}$ provided by (34), $\theta$ is given by (35) and $\epsilon$ is a variable used to model the mean error.

Proof: Same as the proof of Theorem 1

\section{SCN DETECTOR}

The SCN detector is another blind detector that uses the eigenvalues of the sample covariance matrix in decision making [6], [18], [48]-[50]. It is defined as the ratio of the largest to the smallest eigenvalue of the covariance matrix as follows:

$$
X_{S C N}=\frac{\lambda_{1}}{\lambda_{K}}
$$

In literature, the SCN metric was studied asymptotically in [4] and the threshold was presented according to MP law. In [6], the authors improved the accuracy of the asymptotic statistical distribution of the SCN by using the TW distribution. This work was further extended in [16] by using Curtiss formula where both the largest and the smallest eigenvalues converge to TW distributions when $(K, N) \rightarrow+\infty$ as shown in [51], [52].However, all these distributions are not analytically tractable. Hence, a simplification of these distributions is required.

1) Null Hypothesis Case: The SCN metric distribution can be approximated using a simple and accurate GEV PDF given by Theorem 3 [19]:

Theorem 3. Let $X_{S C N}$ be the statistical metric associated to $\boldsymbol{W} \sim \mathcal{C W}_{K}\left(N, \sigma_{b}^{2} \boldsymbol{I}_{K}\right)$. If AC condition is satisfied, then the $C D F$ and PDF of $X_{S C N}$ can be asymptotically and tightly approximated respectively by:

$$
\begin{aligned}
& F\left(x ; \theta_{0}, \beta_{0}, \xi_{0}\right)=e^{-\left(1+\left(\frac{x-\theta_{0}}{\beta_{0}}\right) \xi_{0}\right)^{-1 / \xi_{0}}} \\
& f\left(x ; \theta_{0}, \beta_{0}, \xi_{0}\right)=\frac{1}{\beta_{0}}\left(1+\left(\frac{x-\theta_{0}}{\beta_{0}}\right) \xi_{0}\right)^{\frac{-1}{\xi_{0}}-1} e^{-\left(1+\left(\frac{x-\theta_{0}}{\beta_{0}}\right) \xi_{0}\right)^{-1 / \xi_{0}}}
\end{aligned}
$$

where $\xi_{0}, \beta_{0}$ and $\theta_{0}$ are defined respectively by:

$$
\begin{aligned}
\xi_{0} & =-0.06393 \mathcal{S}_{X_{S C N}}^{2}+0.3173 \mathcal{S}_{X_{S C N}}-0.2771 \\
\beta_{0} & =\sqrt{\frac{\sigma_{X_{S C N}}^{2} \xi^{2}}{g_{2}-g_{1}^{2}}} \\
\theta_{0} & =\mu_{X_{S C N}}-\frac{\left(g_{1}-1\right) \beta}{\xi}
\end{aligned}
$$

where $\mu_{X_{S C N}}, \sigma_{X_{S C N}}^{2}$ and $\mathcal{S}_{X_{S C N}}$ are defined in $[19$, Theorem 1] and $g_{i}=\Gamma(1-i \xi)$.

Proof: Refer to [19] for the detailed proof.

2) $\mathcal{H}_{1}$ Hypothesis Case: Likewise, we introduce the following theorem which approximates the distribution of $X_{S C N}$ using the simple GEV distribution under $\mathcal{H}_{1}$ hypothesis.

Theorem 4. Let $X_{S C N}$ be the statistical metric associated to $\boldsymbol{W} \sim \mathcal{C W}_{K}(N, \boldsymbol{\Sigma})$. If $A C$ and $C C$ conditions are satisfied, then the CDF and PDF of $X_{S C N}$ can be asymptotically and tightly approximated by:

$F\left(x ; \theta_{1}, \beta_{1}, \xi_{1}\right)=e^{-\left(1+\left(\frac{x-\theta_{1}}{\beta_{1}}\right) \xi_{1}\right)^{-1 / \xi_{1}}}$

$f\left(x ; \theta_{1}, \beta_{1}, \xi_{1}\right)=\frac{1}{\beta_{1}}\left(1+\left(\frac{x-\theta_{1}}{\beta_{1}}\right) \xi_{1}\right)^{\frac{-1}{\xi_{1}}-1} e^{-\left(1+\left(\frac{x-\theta_{1}}{\beta_{1}}\right) \xi_{1}\right)^{-1 / \xi}}$ where $\xi_{1}, \beta_{1}$ and $\theta_{1}$ are defined respectively by:

$$
\begin{aligned}
\xi_{1} & =-0.06393 \mathcal{S}_{X_{S C N}}^{2}+0.3173 \mathcal{S}_{X_{S C N}}-0.2771 \\
\beta_{1} & =\sqrt{\frac{\sigma_{X_{S C N}}^{2} \xi^{2}}{g_{2}-g_{1}^{2}}} \\
\theta_{1} & =\mu_{X_{S C N}-\frac{\left(g_{1}-1\right) \beta}{\xi}}
\end{aligned}
$$

where $\mu_{X_{S C N}}, \sigma_{X_{S C N}}^{2}$ and $\mathcal{S}_{X_{S C N}}$ are defined in $[19$, Theorem 2] and $g_{i}=\Gamma(1-i \xi)$.

Proof: Refer to [19] for the detailed proof.

\section{FAE AND PAE: PERFORMANCE PROBABILITIES}

The target of this section is to summarize the different cases analyzed in this paper and provide the corresponding performance metrics given here in terms of $P_{f a}$ and $P_{d}$. The latter will be used in the threshold optimization and the system specifications within the ELASTIC framework.

\section{A. Summary On the Proposed Analytical Derivations}

It is very clear from the previous sections that the proposed yet simple approximations could be directly applied within the framework of ELASTIC. For the sake of simplicity, we give in Table I, a summary of the proposed approximations. They will be used in the optimization of the CR system metrics in both the PAE and FAE scenarios with any transmission conditions.

\section{B. Performance Probabilities of the LE Detector}

In this section, we present the two mentioned scenarios of antenna exploitation within the ELASTIC framework. In the FAE scenario, the CR will use all of its antennas for the SS process. Accordingly, the LE detector will be working in the asymptotic regime of both $K$ and $N$. On the other hand, in the PAE scenario the CR will use a fewer number of antennas for the SS process and thus the LE detector will be working in the asymptotic regime of $N$ only (i.e. $N$ is relatively large w.r.t $K$ ).

1) FAE Scenario: According to Proposition 1 and using (8), the expression of $P_{f a}$ is given by:

$$
P_{f a}=1-e^{\left.-\left(1+\left(\frac{\hat{\lambda}_{X_{L E}}-\theta}{\beta}\right) \xi\right)^{-1 / \xi}\right)} .
$$

Similarly, by using (16) and (9) the expression of $P_{m d}$ is given by:

$$
P_{m d}=\Phi\left(\frac{\hat{\lambda}_{X_{L E}}-\mu_{1}}{\sigma_{1}}\right)
$$

where $\Phi$ is the CDF of the standard normal distribution, $\hat{\lambda}_{X_{L E}}$ is the threshold of the LE detector, $\mu_{1}$ and $\sigma_{1}$ are respectively the mean and the standard deviation of the LE metric under $\mathcal{H}_{1}$ given in (16). 
TABLE I

SUMMARY OF THE PROPOSED PDFs

\begin{tabular}{|c||l|l||l|l|}
\hline \hline Metric & Existing Dist. (under $\mathcal{H}_{0}$ ) & Existing Dist. (under $\mathcal{H}_{1}$ ) & Proposed Dist. (under $\mathcal{H}_{0}$ ) & Proposed Dist. (under $\mathcal{H}_{1}$ ) \\
\hline LE & TW2 or Normal & Normal & Proposition 1 - GEV & Normal \\
\hline SLE & using TW2 or Mellin Trans. & using Mellin Trans. & Theorem 1 - Gaussian function & Theorem 2 - Gaussian function \\
\hline SCN & TW2 with Curtiss Formula & TW2 with Curtiss Formula & Theorem 3 - GEV & Theorem 4 - GEV \\
\hline
\end{tabular}

2) PAE Scenario: Here, we consider the following:

- FPAE approach: in this case, the CR will use certain fixed number $K$ and $N>>K$.

- DPAE approach: here, the CR will use dynamic number of antenna $K$ and $N>>K$.

In both cases, $K$ is finite and $N$ is relatively large. Under $\mathcal{H}_{0}$ hypothesis, $X_{L E}$ is approximated by Normal distribution where the mean and the variance are approximated using (19) and (20) respectively. Under $\mathcal{H}_{1}$ hypothesis, results in [42] show that $X_{L E}$ could be approximated by Gaussian distribution in (16) even for small values of $K$. Then, $P_{f a}$ and $P_{m d}$ are expressed as follows:

$$
\begin{aligned}
P_{f a} & =1-\Phi\left(\frac{\hat{\lambda}_{X_{L E}}-\mu_{0}}{\sigma_{0}}\right) \\
P_{m d} & =\Phi\left(\frac{\hat{\lambda}_{X_{L E}}-\mu_{1}}{\sigma_{1}}\right)
\end{aligned}
$$

where $\Phi($.$) is the CDF of the standard normal distribution; \mu_{0}$ and $\sigma_{0}$ are the mean and the standard deviation of $X_{L E}$ under $\mathcal{H}_{0}$ hypothesis and are given by (19) and (20) respectively; $\mu_{1}$ and $\sigma_{1}$ are the mean and the standard deviation of the $X_{L E}$ under $\mathcal{H}_{1}$ hypothesis and are given by (17) and (18).

\section{Performance Probabilities of the SLE and SCN Detectors} by:

Using (8) and (28), the $P_{f a}$ of the SLE detector is given

$$
P_{f a}(\alpha)=Q\left(\frac{\hat{\lambda}_{X_{S L E}} \mu_{T_{n}}-\mu_{\lambda_{1}}}{\sqrt{\sigma^{2} \lambda_{1}-2 \alpha c+\alpha^{2} \sigma^{2} T_{n}}}\right)
$$

where $Q($.$) is the Q-function. \mu_{\lambda_{1}}, \sigma^{2}{ }_{\lambda_{1}}, \mu_{T_{n}}$ and $\sigma^{2} T_{n}$ are given respectively (19), (20), (31) and (32). $P_{m d}$ is derived the same way using $\mathcal{H}_{1}$ hypothesis. Using Theorems 3 and 4 , and using (8) and (9), the $P_{f a}$ and $P_{m d}$ of the SCN detector are respectively expressed as:

$$
\begin{aligned}
& P_{f a}=1-e^{-\left(1+\left(\frac{\hat{\lambda}_{X_{S C N}}-\theta_{0}}{\beta_{0}}\right) \xi_{0}\right)^{-1 / \xi_{0}},} \\
& P_{m d}=1-P_{d}=e^{-\left(1+\left(\frac{\lambda_{X_{S C N}}-\theta_{1}}{\beta_{1}}\right) \xi_{1}\right)^{-1 / \xi_{1}} .}
\end{aligned}
$$

\section{THRESHOLD OPTIMIZATION}

The target of this section is to derive the optimal decision threshold for a desired $P_{f a}$ and/or $P_{m d}$. However, as these metrics depend on $K, N$, and maybe the SNR (in case of LE), the optimization of this threshold will depend on these main parameters. Hence, finding the optimal values of $K, N$ for a target $P_{f a}$ and/or $P_{m d}$ is analytically not straightforward. To do so, the designer has to select a key performance or run an algorithmic search as shown in Algorithm 2. In both cases, the optimization is still easier than using of the exact distribution (such TW2) of the sensing metric or using the LUTs. Definitely, if the system parameters are to be changed dynamically (i.e. DPAE), then the optimization problem turns out to be finding the optimal value of $K$ and/or $N$ that fit the performance requirements. It is worth mentioning that the execution time of Algorithm 2 is too small in our simulations. Despite this fact, the search of the optimal solution could be made faster by specifying a range of value for $K$ and $N$. In this section, the optimal threshold is given for a given $K$ and $N$.

\section{A. Threshold Optimization for the LE Detector}

The performance probabilities depend on the decision threshold $\left(\hat{\lambda}_{X_{L E}}\right)$, hence it is necessary to choose an appropriate value based on system requirements. The typical approach for setting the threshold is given by the constant false-alarm rate (CFAR) strategy in which the threshold is chosen in order to guarantee a target false-alarm rate $\left(\hat{p}_{f a}\right)$. Based on the CFAR scenario, then the decision threshold is expressed using the inverse of $P_{f a}$ as follows:

1) FAE Scenario:

$$
\hat{\lambda}_{X_{L E}}=\mu+\frac{\sigma}{\xi}\left(-1+\left[-\ln \left(1-\hat{P}_{f a}\right)\right]^{-\xi}\right)
$$

2) PAE Scenario: For finite $K$ and relatively large $N$ and based on the CFAR scenario, the decision threshold is expressed using the inverse of $P_{f a}$ as follows:

$$
\hat{\lambda}_{X_{L E}}=\mu_{0}+\sigma_{0} \Phi^{-1}\left(1-\hat{P}_{f a}\right)
$$

The CFAR threshold selection strategy is not optimal since it ensures a fixed false-alarm probability rather than a required SS performance given in terms of both $P_{f a}$ and $P_{m d}$. An optimal threshold could be selected as to minimize the total error probability of the system such that:

$$
\hat{\lambda}_{X_{L E}}=\underset{\alpha}{\operatorname{argmin}}\left(w_{0} P_{f a}+w_{1} P_{m d}\right)
$$

where $w_{0}$ and $w_{1}$ are weighting coefficients that are chosen according to system priority. In order to solve this minimization problem, one can simply take the derivative equals to zero and the second derivative positive (i.e. concave). One can choose $w_{0}=0$ or $w_{1}=0$ to minimize one of the error probabilities. However, it is typical to choose $w_{0}=w_{1}=0.5$ to minimize the sum of the error probabilities. Then $\lambda_{X_{L E}}$ should be selected such that it minimizes $P_{f a}+P_{m d}$, i.e. its derivative is equal to zero:

$$
\left(\frac{1}{2 \sigma_{0}^{2}}-\frac{1}{2 \sigma_{1}^{2}}\right) \lambda_{X_{L E}}^{2}+\left(\frac{\mu_{1}}{\sigma_{1}^{2}}-\frac{\mu_{0}}{\sigma_{0}^{2}}\right) \lambda_{X_{L E}}+\left(\frac{\mu_{0}^{2}}{2 \sigma_{0}^{2}}-\frac{\mu_{1}^{2}}{2 \sigma_{1}^{2}}-\ln \left(\frac{w_{0} \sigma_{1}}{w_{1} \sigma_{0}}\right)\right)=0
$$


(62):

The optimal threshold is then given by finding the roots of

$\hat{\lambda}_{X_{L E}}=\frac{\mu_{1} \sigma_{0}^{2}-\mu_{0} \sigma_{1}^{2}+\sqrt{\sigma_{0}^{2} \sigma_{1}^{2}\left(\left(\mu_{0}-\mu_{1}\right)^{2}-2\left(\sigma_{0}^{2}-\sigma_{1}^{2}\right) \ln \left(\frac{w_{0} \sigma_{1}}{w_{1} \sigma_{0}}\right)\right)}}{\sigma_{0}^{2}-\sigma_{1}^{2}}$

\section{Observations on (63):}

- The optimal threshold in (63) requires the knowledge of the SNR value.

- The weights $w_{0}$ and $w_{1}$ could be tuned according to the required $P_{f a}$ and $P_{m d}$. A reduced $w_{0}$ means an improved $P_{f a}$ which requires a larger $\hat{\lambda}_{X_{L E}}$. This is in line with (63). The same conclusion could be derived on $w_{1}$.

- It could be used in any of the scenarios to minimize the error probabilities. However, in the dynamic case, i.e. when $K$ and/or $N$ are to be selected dynamically, the designer should set up the target $P_{f a}$ and $P_{m d}$ to be obtained while the optimization will consist in finding the minimal $K$ and $N$. Accordingly, a fixed $\left(\hat{P}_{f a}, \hat{P}_{d}\right)$ is selected to evaluate the required $K$ and $N$ to achieve this performance. This will be discussed next.

\section{B. Minimum Requirements For the LE Detector with Dynamic Parameters}

For a target $\left(\hat{P}_{f a}, \hat{P}_{d}\right)$ and at a given SNR $\rho$, the CR system should optimize certain number of antennas for a certain number of samples. By eliminating $\lambda_{X_{L E}}$ from $P_{f a}$ and $P_{d}$ in (54) and (55) respectively, one can solve for $K$ (or $N$ ) the following equation:

$$
\sigma_{0} \Phi^{-1}\left(1-\hat{P}_{f a}\right)-\sigma_{1} \Phi^{-1}\left(1-\hat{P}_{d}\right)+\mu_{0}-\mu_{1}=0
$$

Hence, at a certain SNR value and for a target detection performance $\left(\hat{P}_{f a}, \hat{P}_{d}\right)$ the system can dynamically choose the couple $(K, N)$ that most enhances its global performance (i.e. throughput, power saving etc.). Note that finding a general solution for (64) is not straightforward and thus we solve for numerical values. Example 1: Consider the following example, $\hat{P}_{f a}=0.1, \hat{P}_{d}=0.9$ and $\rho=-15 d B$, then we get Tables II and III.

TABLE II

REQUiRED $K$ FOR A GIVEN $N$ IN EXAMPLE 1.

\begin{tabular}{|l||l|l|l|l|l|l|l|l|}
\hline \hline $\mathrm{N}$ & 200 & 300 & 350 & 400 & 450 & 500 & 600 & 1000 \\
\hline $\mathrm{K}$ & 14 & 10 & 8 & 8 & 7 & 6 & 5 & 4 \\
\hline
\end{tabular}

TABLE III

REQUIRED $N$ FOR A GIVEN $K$ IN EXAMPLE 1.

\begin{tabular}{|l||l|l|l|l|l|}
\hline \hline $\mathrm{K}$ & 20 & 18 & 15 & 10 & 8 \\
\hline $\mathrm{N}$ & 132 & 147 & 177 & 273 & 349 \\
\hline
\end{tabular}

Table II provides, for each value of $N$, the corresponding number $K$ of antennas required in the sensing process to achieve the target performance. On the other hand, Table III provides for each value of $K$ the corresponding number of samples $N$ that should be acquired by each antenna to achieve the considered performance. It is worth mentioning that the values of $K$ and $N$ evaluated using (64) are real valued numbers and thus are rounded to $+\infty$. The designer can choose for instance $K=8$ and $N=350$ configuration or $K=6$ and $N=500$. The system may also be expected to have dynamic behavior in case of a change in the SNR values. This could be also achieved using (64).

\section{Extension to SLE and SCN Detectors}

This section extends the dynamic antenna exploitation on the LE detector and finds the minimum requirements for both SLE and SCN detectors within ELASTIC framework.

1) SCN Detector: The threshold could be computed using (57) and (58) according to a required error constraint. For example, for a target $P_{f a}$, the threshold is given by:

$$
\hat{\lambda}_{X_{S C N}}=\theta_{0}+\frac{\beta_{0}}{\xi_{0}}\left(-1+\left[-\ln \left(1-P_{f a}\right)\right]^{-\xi_{0}}\right) .
$$

For a target $\left(\hat{P}_{f a}, \hat{P}_{d}\right)$, by considering the SCN detector and eliminating $\lambda_{X_{S C N}}$ from both (57) and (58) we get:

$\theta_{0}-\frac{\beta_{0}}{\xi_{0}}-\theta_{1}+\frac{\beta_{1}}{\xi_{1}}+\frac{\beta_{0}}{\xi_{0}}\left[-\ln \left(1-\hat{P}_{f a}\right)\right]^{-\xi_{0}}-\frac{\beta_{1}}{\xi_{1}}\left[-\ln \left(1-\hat{P}_{d}\right)\right]^{-\xi_{1}}=0$

where $\theta_{i}, \beta_{i}$ and $\xi_{i}$ are the location, scale and shape parameters of the GEV distribution where $i=0$ refers to $\mathcal{H}_{0}$ hypothesis and $i=1$ refers to $\mathcal{H}_{1}$ hypothesis. Their expressions are provided by Theorems 3 and 4 .

2) SLE Detector: Here, same derivations are provided. Hence, we can write:

$$
\begin{aligned}
& \frac{\mu_{12}^{\mathcal{H}_{0}}-\Delta^{2} r_{0} \sigma_{12}^{\mathcal{H}_{0}}}{\mu_{T_{n}^{\mathcal{H}_{0}}}^{2}-\Delta^{2} \sigma_{T_{n}^{2}}^{2}}-\frac{\mu_{12}^{\mathcal{H}_{0}}-\Lambda^{2} r_{1} \sigma_{12}^{\mathcal{H}_{1}}}{\mu_{T_{n}^{\mathcal{H}_{1}}}^{2}-\Lambda^{2} \sigma_{T_{n}^{\mathcal{H}_{1}}}^{2}} \\
& +\frac{\Delta \sqrt{m_{v}^{\mathcal{H}_{0}}-2 r_{0} \mu_{12}^{\mathcal{H}_{0}} \sigma_{12}^{\mathcal{H}_{0}}+\Delta^{2}\left[\sigma_{12}^{\mathcal{H}_{0}}\right]^{2}\left(r_{0}^{2}-1\right)}}{\mu_{T_{n}^{\mathcal{H}_{0}}}^{2}-\Delta^{2} \sigma_{T_{n}^{2}}^{2} \mathcal{H}_{0}} \\
& -\frac{\Lambda \sqrt{m_{v}^{\mathcal{H}_{1}}-2 r_{1} \mu_{12}^{\mathcal{H}_{1}} \sigma_{12}^{\mathcal{H}_{1}}+\Lambda^{2}\left[\sigma_{12}^{\mathcal{H}_{1}}\right]^{2}\left(r_{1}^{2}-1\right)}}{\mu_{T_{n}^{\mathcal{H}_{1}}}^{2}-\Lambda^{2} \sigma_{T_{n}^{\mathcal{H}_{1}}}^{2}}=0
\end{aligned}
$$

where $\Delta=Q^{-1}\left(\hat{P}_{f a}\right)$ and $\Lambda=Q^{-1}\left(\hat{P}_{d}\right)$ with $Q^{-1}($.$) is$ the inverse Q-function; the expressions $\mu_{12}^{\mathcal{H}_{i}}=\mu_{\lambda_{1}^{\mathcal{H}_{i}}} \mu_{T_{n}^{\mathcal{H}_{i}}}$, $\sigma_{12}^{\mathcal{H}_{i}}=\sigma_{\lambda_{1}^{\mathcal{H}_{i}}} \sigma_{T_{n}^{\mathcal{H}_{i}}}, m_{v}^{\mathcal{H}_{i}}=\mu_{T_{n}^{\mathcal{H}_{i}}}^{2} \sigma_{\lambda_{1}^{\mathcal{H}_{i}}}^{2}+\mu_{\lambda_{1}^{\mathcal{H}_{i}}}^{2} \sigma_{T_{n}^{\mathcal{H}_{i}}}^{2}$ and $\mathcal{H}_{i}$ refers to one of the two hypothesis $\mathcal{H}_{0}$ and $\mathcal{H}_{1}$ under which the expressions are calculated. Similar to the LE detector case, using (66) and (67) we can determine the minimum requirements of the system that could be used to achieve a target performance.

\section{Simulation And Discussion}

In this section, we verify the analytical derivation results through Monte-Carlo simulations. We validate the theoretical analysis presented in sections IV-B through VIII-C. The simulation results are obtained by generating $10^{5}$ random realizations of $\boldsymbol{Y}$. 


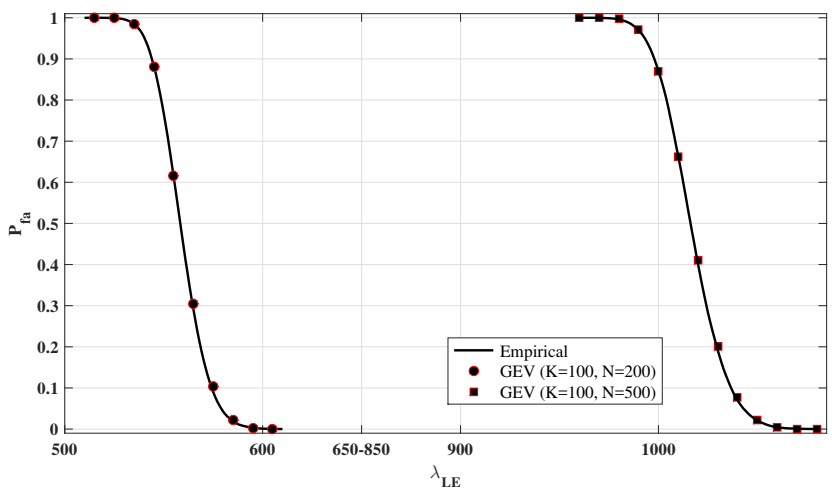

Fig. 1. Empirical $P_{f a}$ of the LE detector in the asymptotic case and its corresponding GEV approximation for $K=100$ and different values of $N$.

\section{A. Validation of Analytical Results}

The asymptotic approximation provided by Proposition 1 is validated in Fig. 1. The results are taken for two different values of $N$ while fixing $K=100$ and $\rho=-10 \mathrm{~dB}$. The results show a perfect match between empirical results and the proposed approximation.

\section{B. Full and Partial antenna exploitation scenarios}

Figure 2 shows the empirical $P_{f a}$ and $P_{d}$ of the LE detector and its corresponding target values $\left(\hat{P}_{f a}=0.01, \hat{P}_{d}=0.9\right)$ while changing the other parameters. In Fig. 2(a) we set $\rho=-20 d B$ and we consider a variable $N$ while in Fig. 2(b) we set $N=500$ and we consider a variable $\rho$, as summarized by Algorithm 2. Simulation results show high accuracy of the analytical results evaluated using (64). The empirical $P_{f a}$ is indeed 0.01 while the accuracy of the $P_{d}$ increases as $K$ increases which reflects the effect of AC under $\mathcal{H}_{1}$ hypothesis. The very small difference between the empirical and the target performance is due to the rounding of $K$ to $+\infty$. The effect of rounding of $K$ could be clearly noticed in Fig. 2(b) for $\rho=-12 d B$ where the exact value is $K=3.3$ and the rounded value is $K=4$. Moreover, the results show that as $N$ or $\rho$ increases the number of antennas required to achieve the target performance decreases and hence these antennas could be exploited for other use.

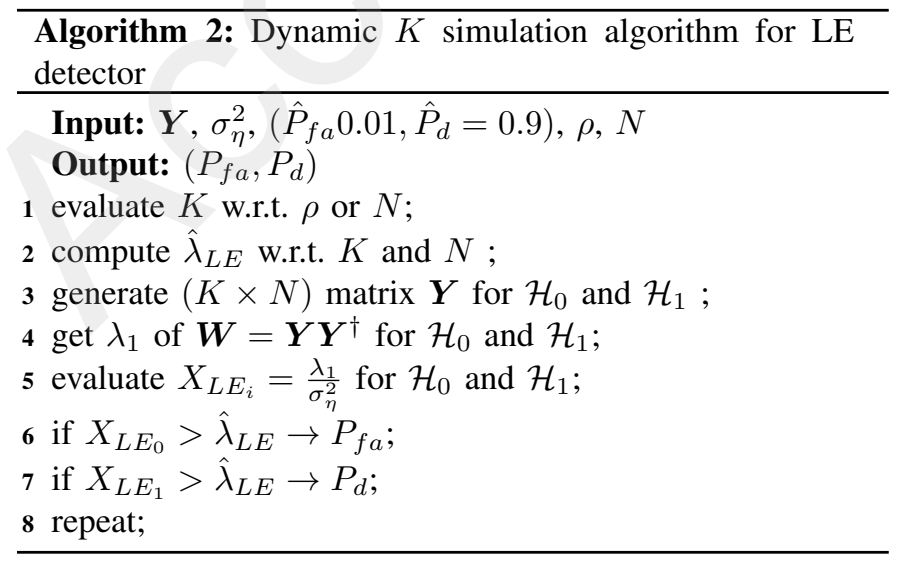

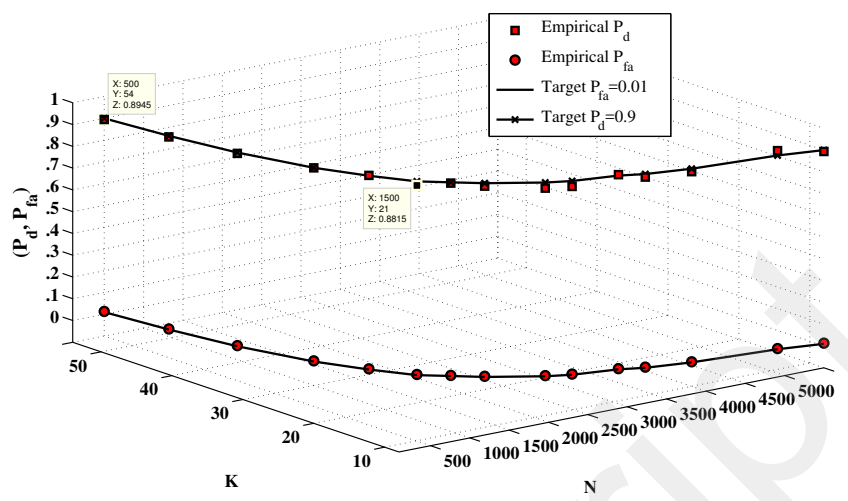

(a) Fixed $\rho=-20 d B$ and variable $N$

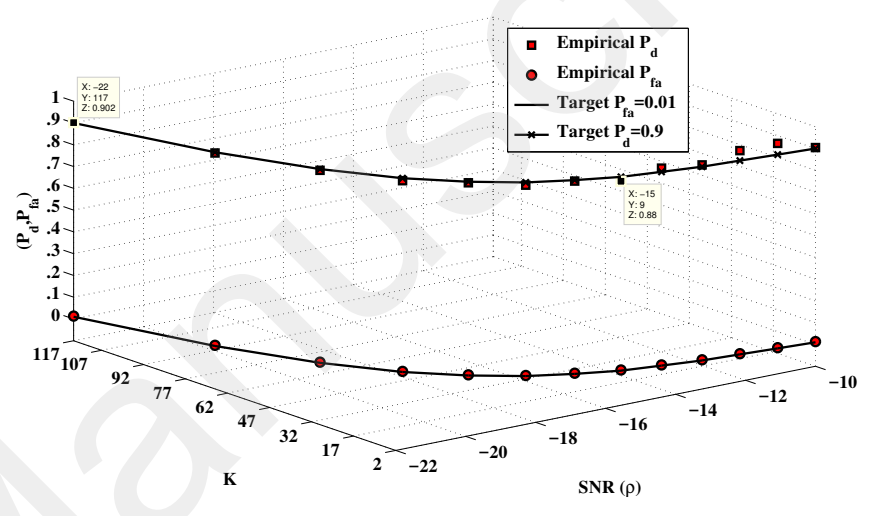

(b) Fixed $N=500$ and variable $\rho$

Fig. 2. Empirical $P_{f a}$ and $P_{d}$ of the LE detector and its corresponding target values by using dynamic method.

Figure 3 shows a comparison between different scenarios, full, dynamic and fixed antenna exploitation. Therein, we consider a CR with $K=200$ antennas. In the case of FPAE, only 5 antennas are used while $K$ is dynamic in DPAE. In the latter, the value of $K$ is calculated using (64) and depends on the SNR. It is obvious in this figure that the full exploitation of the antenna scenario achieves the best performance, however it is exploiting all the antennas all the time even if it not necessary. In this case, the threshold is calculated using the GEV approximation in Sec. IV-A. The use of a fixed number of antennas leads to a worst performance as the transmission conditions get worse. However, if the target performance is well defined then it can be achieved all the time through an efficient use of the antennas. From the figure, the results show a tremendous decrease in the value of $K$ as the SNR increases with approximately stable $P_{d}=0.9$. Using dynamic exploitation scenario, the CR system will gain around 115 antennas that could be used for other purposes. In addition, the computational complexity of the sample covariance matrix and the eigenvalues in the detection algorithm will be decreased since the received matrix size, $(K \times N)$, is decreased. Moreover, results show that it is enough to use $K=2$ for $N=500$ starting from $\rho=-10 d B$. Indeed, since $K=2$ is the smallest value for the EBD then, for $\rho \leq-10 d B$, the designer can fix $K=2$ and starts to minimize $N$ accordingly. In this case, as $N$ decreases the 
threshold could be computed using the GEV approximation in the finite case (see Table I).

The SCN and SLE detectors are considered next using similar approach as in Algorithm 2. The results are shown in Figures 4 through 7. Figures 4 and 6 show $P_{d}$ and $P_{f a}$ of the SCN and SLE detectors respectively for different values of $K$. The latter is changed according to the target $\left(\hat{P}_{f a}=0.01, \hat{P}_{d}=0.9\right)$ and, the variation of SNR or $N$. Figures 5 and 7 show the variation of $P_{d}$ with respect to SNR and $K$ in the aforementioned scenarios. In the SLE detector case, we suppose that the mean error $\epsilon=0$. Results show high accuracy of the analytical results evaluated using (66) and (67). Like the LE case, when $K$ takes small values, the negligible difference between the empirical and target $P_{d}$ is mainly due the rounding of $K$. Moreover, it could be noticed from Fig. 5 that at $\rho=-22 d B$ and using $N=500$ then the required value of $K$ is 203. In this case and since the CR is equipped with 200 antennas, then the designer must fix $K=200$ and starts to increase $N$ accordingly to achieve the target performance. For larger $\rho$, these expressions are very useful and accurate to make a dynamic system in which the antennas are efficiently utilized.

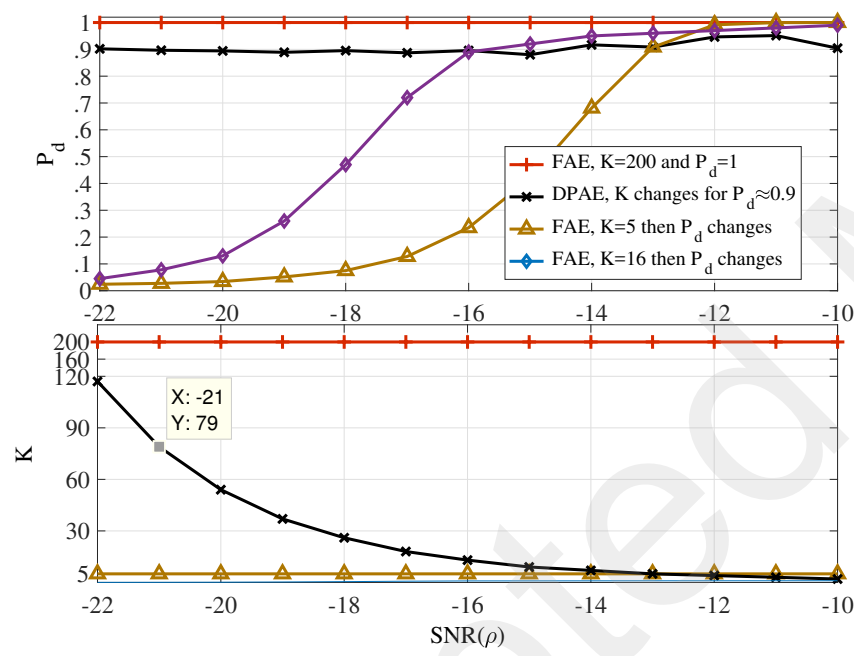

Fig. 3. Empirical $P_{d}$ of the LE detector and the corresponding number of antennas, $K$, used for sensing in Full, Dynamic and Fixed methods w.r.t $\rho$ and fixing $N=500$.

\section{LE, SCN and SLE comparison}

In this section, we provide a comparison between the considered detectors. For the LE detector, the noise power is supposed to be perfectly known while SCN and SLE detectors are totally-blind and do not require this knowledge. Figure 8 plots the Region of Convergence (ROC) of these detectors for $N=500, K=5$ and $\rho=-15 d B$. Simulation results show that the LE detector outperforms the SLE detector and the SLE detector in turn outperforms the SCN detector. Indeed, LE is the optimal detector when noise variance is perfectly known while SLE detector is the optimal under the generalized likelihood ratio (GLR) criterion when noise variance is unknown [7], [10]. However, if noise power is not perfectly known, the performance of the LE detector will

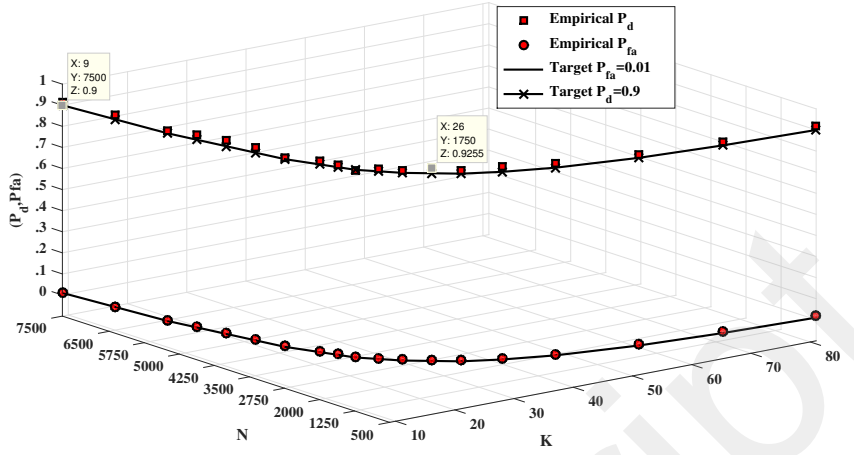

(a) Fixed $\rho=-20 d B$ and variable $N$

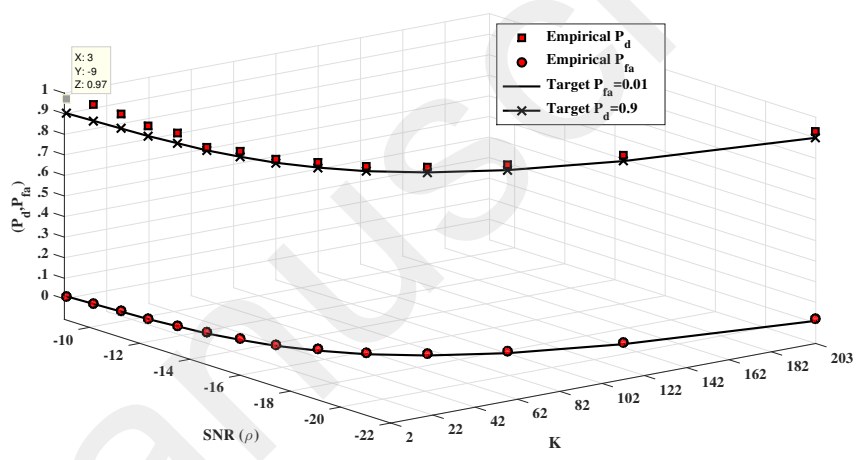

(b) Fixed $N=500$ and variable $\rho$

Fig. 4. Empirical $P_{f a}$ and $P_{d}$ of the SCN detector and its corresponding target values by using dynamic method.

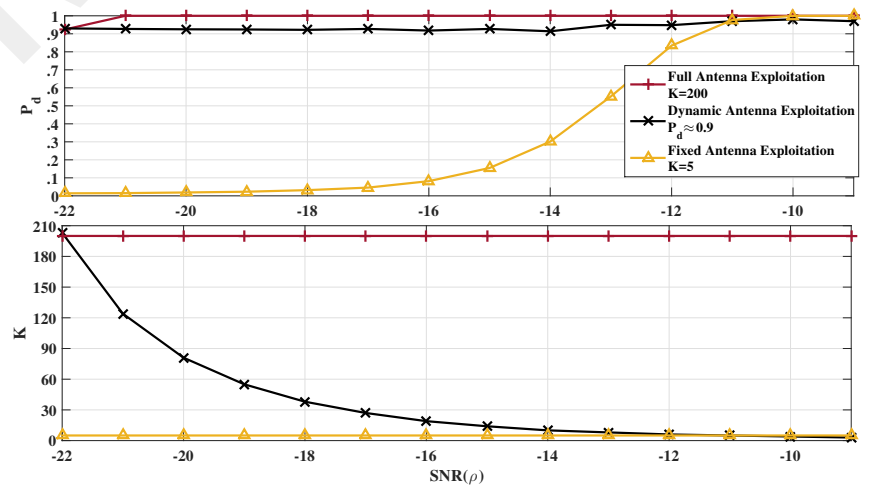

Fig. 5. Empirical $P_{d}$ of the SCN detector and the corresponding number of antennas, $K$, used for sensing in Full, Dynamic and Fixed methods w.r.t $\rho$ and fixing $N=500$.

degrade and it might be even worse than the performance of SLE and SCN detectors as shown in Fig. 8 where $0.2 d B$ noise variance uncertainty is considered.

Figures 9 and 10 show the minimum required number of antennas $K$ for the LE, SCN and SLE detectors to achieve a target $\left(\hat{P}_{f a}=0.01, \hat{P}_{d}=0.9\right)$ when changing $\rho$ and $N$ respectively. Thee results are aligned with those of 9 . In addition, it is also noticeable that the difference in $K$ for LE and SLE are close whereas $K$ required of the SCN detector is larger. Indeed, LE and SLE detectors are optimal when the noise power is perfectly known and noise power uncertain environments respectively. In this regard, it is worth men- 


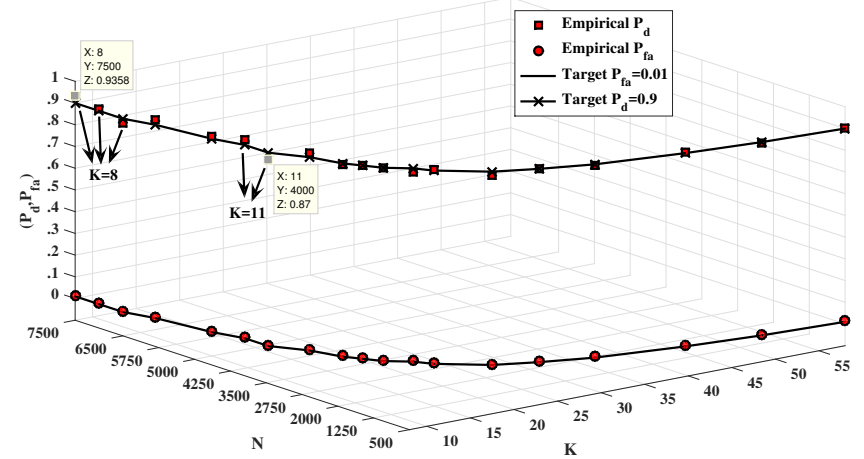

(a) Fixed $\rho=-20 d B$ and variable $N$

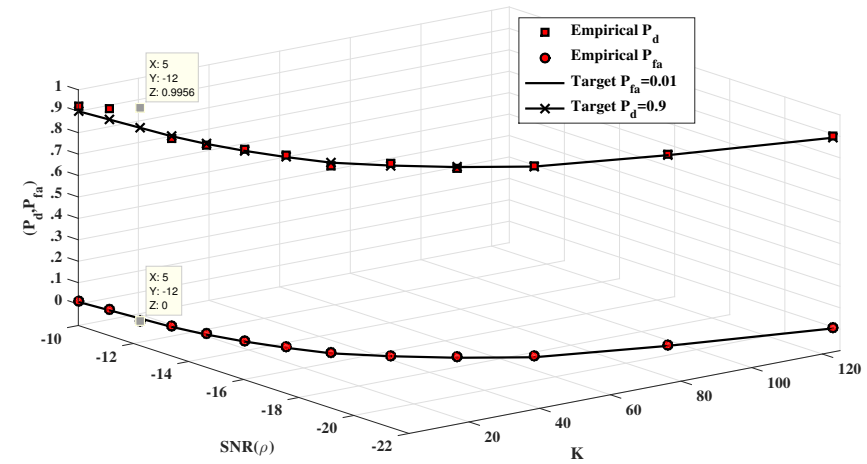

(b) Fixed $N=500$ and variable $\rho$

Fig. 6. Empirical $P_{f a}$ and $P_{d}$ of the SLE detector and its corresponding target values by using dynamic method.

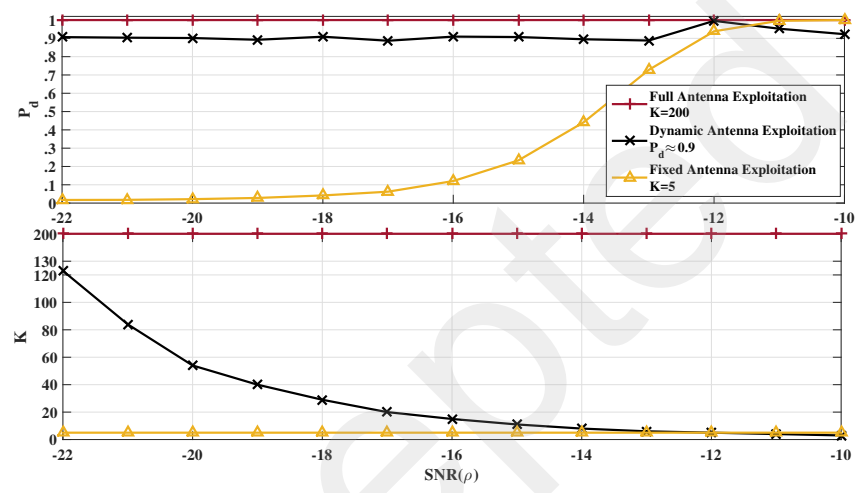

Fig. 7. Empirical $P_{d}$ of the SLE detector and the corresponding number of antennas, $K$, used for sensing in Full, Dynamic and Fixed methods w.r.t $\rho$ and fixing $N=500$.

tioning that by considering LE detector with noise uncertain environment its performance will degrade and thus the number $K$ of required antennas will increase.

\section{Experimental Validation}

The target of this section is to provide some experimental results done in a laboratory environment. In our experiment, we decided to adopt an off-the-shelf prototyping hardware, called Universal Software Radio Peripheral (USRP), provided by National Instruments to design a multi-antenna system. However, as the number of available devices is limited to

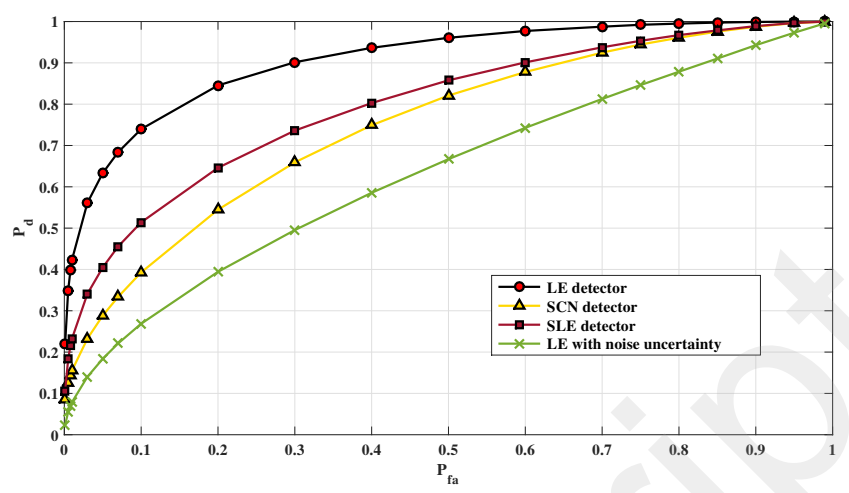

Fig. 8. ROC of the LE, SCN and SLE detectors when $k=5, N=500$ and $\rho=-15 d B$.

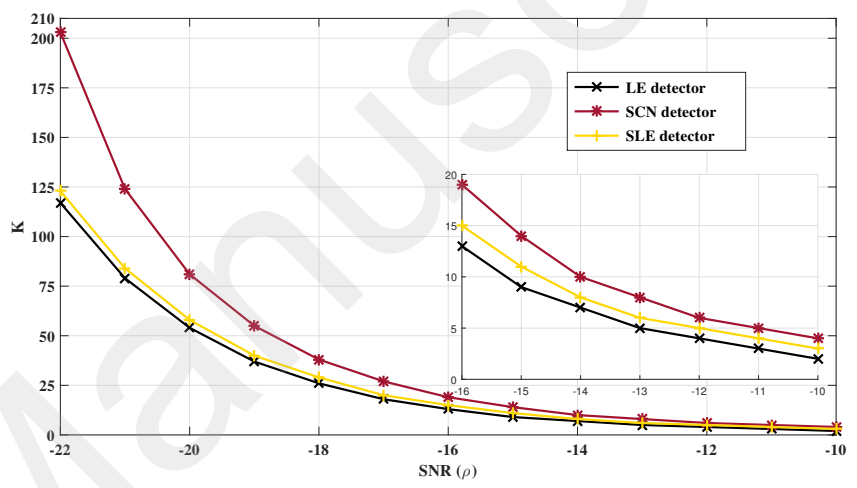

Fig. 9. Required $K$ of the LE, SCN and SLE detectors for dynamic antenna exploitation w.r.t $\rho$ and fixing $N=500, P_{d}=0.9$ and $P_{f a}=0.01$.

6 , the proof-of-concept has been done only in a selected showcase. Moreover, as these devices require synchronization, one USRP should be completely devoted for this purpose. Other synchronization problems (time stamp misalignment, common clock reference) have been also fixed. Finally, one USRP has been selected to emulate the transmitter.

To realize the test bench shown in Fig. 11(a), the following items have been used:

- 4 NI-USRP 2920 used as array elements

- 1 NI-USRP 2920 for synchronization

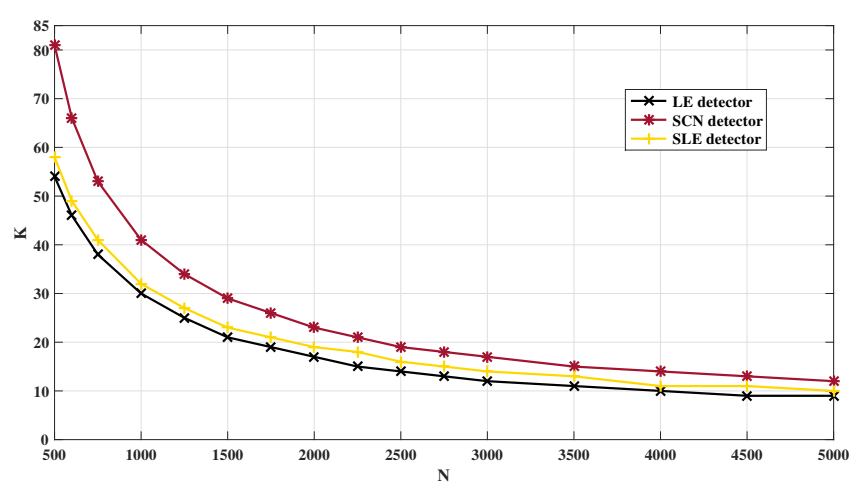

Fig. 10. Required $K$ of the LE, SCN and SLE detectors for dynamic antenna exploitation w.r.t $N$ and fixing $\rho=-20 d B, P_{d}=0.9$ and $P_{f a}=0.01$. 


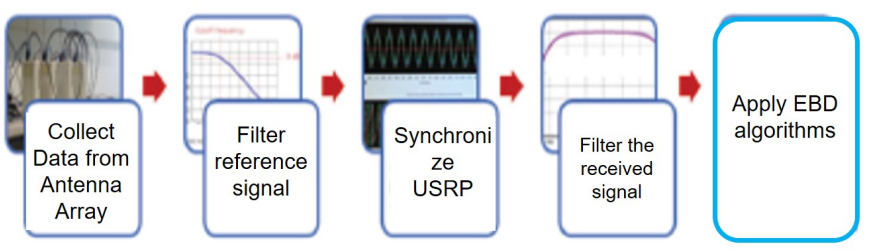

(a) Test Bench Process

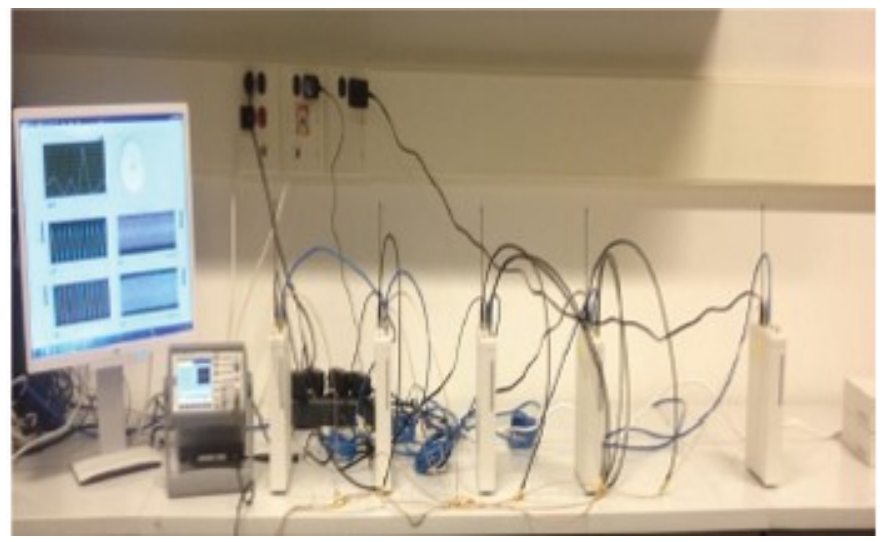

(b) Test Bench Experiment

Fig. 11. Lab Experiment Setup

- 1 NI-USRP 2920 serving as transmitter

- A waveform generator

In this test bench, a $10 \mathrm{MHz}$ sinusoidal signal modulated a signal with carrier frequency of $915 \mathrm{MHz}$. The signal was generated in intermittent times, each of $0.1 \mathrm{sec}$ duration.

Fig. 12 provides the histogram of the SCN measurements under $\mathrm{H} 0$ and H1. It is very clear that the proposed GEV approximation fits very well the SCN measurements. Moreover, GEV approximation outperforms the Gaussian distribution proposed in literature. Finally, Fig.13 shows that the experimental ROC curve is aligned with the theoretical derivations provided in terms of GEV.

\section{CONCLUSiOn}

CR equipped with massive antenna technology will achieve a significant increase in the performance of the multi-antenna SS detector. However, it might be enough to use fewer number of antennas for the sensing process and achieve a desired performance. In this paper, we have considered the LE, SCN and SLE detectors with two exploitation scenarios: FAE and PAE. The latter is further decomposed into two options: (i) fixed use and (ii) dynamic use. We extended our previous work on the approximation of the distribution of the LE detector as a GEV distribution for both finite and asymptotic cases. Then, an optimized decision threshold that minimizes the error probabilities of the LE detector has been derived in both fixed and dynamic cases. Likewise, we used our recent work on SLE and SCN to derive the necessary sensing metrics and design parameters. This paper provided a mathematical framework to compute the minimum requirements of the $\mathrm{CR}$ system to achieve the desired performance of all exploitation scenarios in a massive antennas environment. Finally, it has

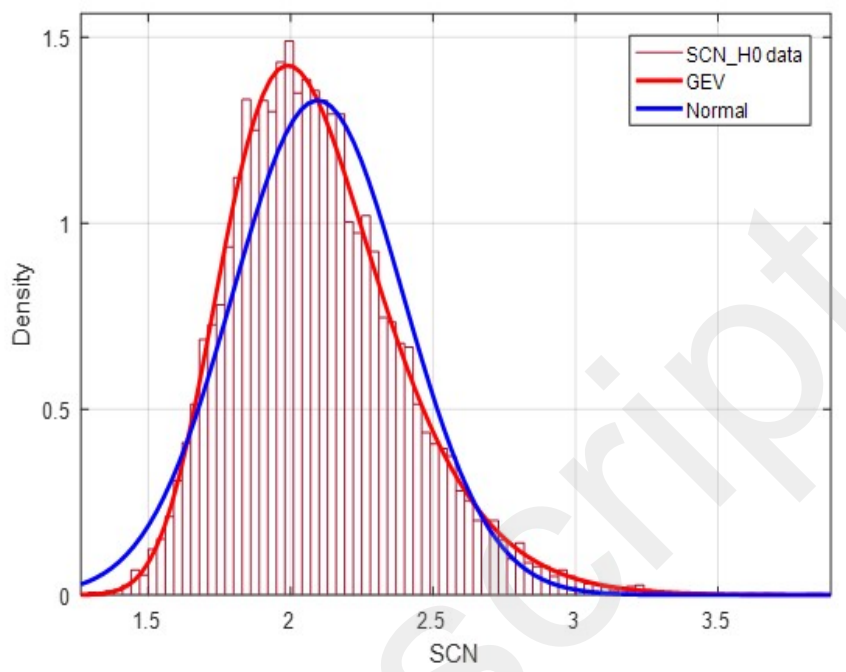

(a) SCN under H0

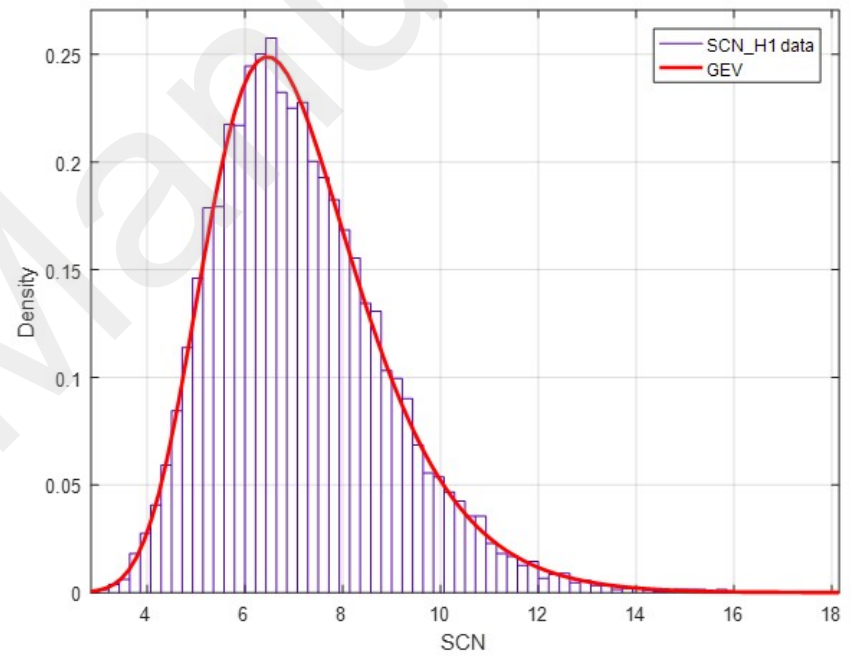

(b) SCN under $\mathrm{H} 1$

Fig. 12. SCN Measurements and PDF Fitting Curves, $\rho=-10 d B, K=4$, $N=500$

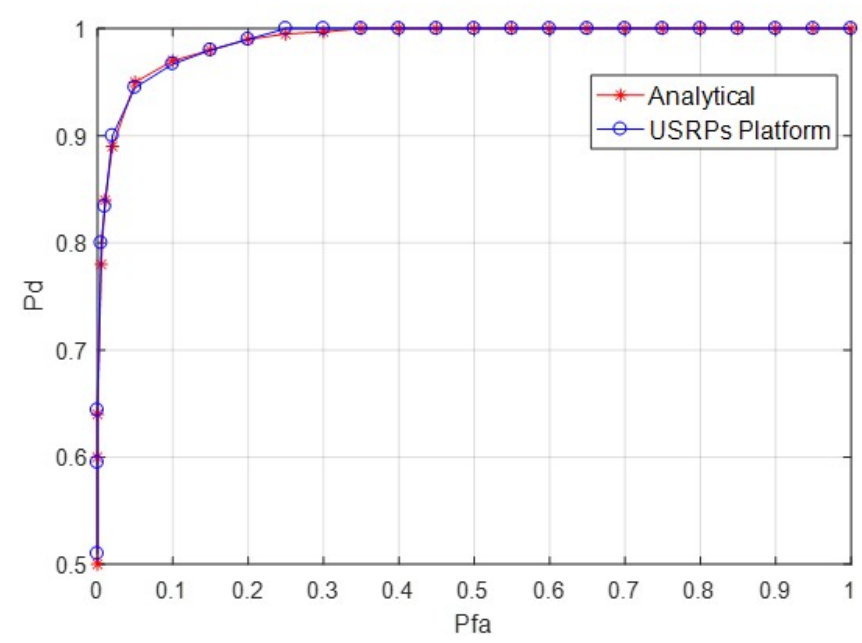

Fig. 13. Experimental SCN ROC Curve 
been shown that the dynamic approach is the best solution for an efficient antenna exploitation. In this case, the CR design parameters should be dynamically tuned to meet the predefined performance and specifications.

\section{REFERENCES}

[1] J. Mitola and G. Q. Maguire, "Cognitive radio: making software radios more personal," IEEE Personal Communications, vol. 6, no. 4, pp. 13 18, Aug 1999.

[2] T. Yucek and H. Arslan, "A survey of spectrum sensing algorithms for cognitive radio applications," IEEE Communications Surveys Tutorials, vol. 11, no. 1, pp. 116-130, 2009.

[3] A. Ali and W. Hamouda, "Advances on spectrum sensing for cognitive radio networks: Theory and applications," IEEE Communications Surveys Tutorials, vol. 19, no. 2, pp. 1277-1304, Secondquarter 2017.

[4] L. Cardoso, M. Debbah, P. Bianchi, and J. Najim, "Cooperative spectrum sensing using random matrix theory," in Proc. IEEE Int. Symp. Wireless Pervasive Comput. (ISWPC), pp. 334-338, May 2008.

[5] Y. Zeng, Y. C. Liang, and R. Zhang, "Blindly combined energy detection for spectrum sensing in cognitive radio," IEEE Signal Processing Letters, vol. 15, pp. 649-652, 2008.

[6] Y. Zeng and Y.-C. Liang, "Eigenvalue-based spectrum sensing algorithms for cognitive radio," IEEE Trans. Commun., vol. 57, no. 6, pp. 1784-1793, June 2009.

[7] B. Nadler, F. Penna, and R. Garello, "Performance of eigenvalue-based signal detectors with known and unknown noise level," Communications (ICC), IEEE International article on, pp. 1-5, June 2011.

[8] B. Nadler, "On the distribution of the ratio of the largest eigenvalue to the trace of a wishart matrix," Journal of Multivariate Analysis, vol. 102, no. 2, pp. 363 - 371, 2011. [Online]. Available: http://www.sciencedirect.com/science/article/pii/S0047259X10002113

[9] P. Bianchi, J. Najim, G. Alfano, and M. Debbah, "Asymptotics of eigenbased collaborative sensing," IEEE Information Theory Workshop (ITW'09), pp. 515-519, Oct 2009.

[10] P. Bianchi, M. Debbah, M. Maida, and J. Najim, "Performance of statistical tests for single-source detection using random matrix theory," IEEE Trans. Inform. Theory, vol. 57, no. 4, pp. 2400-2419, April 2011.

[11] L. Wei, "Non-asymptotic analysis of scaled largest eigenvalue based spectrum sensing," Ultra Modern Telecommunications and Control Systems and Workshops (ICUMT), 2012 4th International Congress on, pp. 955-958, Oct 2012.

[12] L. Wei, M. R. McKay, and O. Tirkkonen, "Exact demmel condition number distribution of complex wishart matrices via the mellin transform," IEEE Communications Letters, vol. 15, no. 2, pp. 175-177, Feb. 2011.

[13] L. Wei, O. Tirkkonen, K. D. P. Dharmawansa, and M. R. McKay, "On the exact distribution of the scaled largest eigenvalue," CoRR, vol. abs/1202.0754, 2012. [Online]. Available: http://arxiv.org/abs/1202.0754

[14] H. Kobeissi, Y. Nasser, A. Nafkha, O. Bazzi, and Y. Louët, "A simple formulation for the distribution of the scaled largest eigenvalue and application to spectrum sensing," CROWNCOM 2016, pp. 284-293, May 2016.

[15] H. Kobeissi, A. Nafkha, Y. Nasser, O. Bazzi, and Y. Louët, "Scaled largest eigenvalue in spectrum sensing: A simple form approach,' EAI Endorsed Transaction on Cognitive Communications, vol. 3, no. 10, 2017.

[16] F. Penna, R. Garello, D. Figlioli, and M. Spirito, "Exact non-asymptotic threshold for eigenvalue-based spectrum sensing," Proc. IEEE 4th Int. Conf. CROWNCOM, pp. 1-5, June 2009

[17] F. Penna, R. Garello, and M. Spirito, "Probability of missed detection in eigenvalue ratio spectrum sensing," Wireless and Mobile Computing, Networking and Communications, 2009. WIMOB 2009. IEEE International article on, pp. 117-122, Oct 2009.

[18] W. Zhang, G. Abreu, M. Inamori, and Y. Sanada, "Spectrum sensing algorithms via finite random matrices," IEEE Trans. Commun., vol. 60, no. 1, pp. 164-175, January 2012.

[19] H. Kobeissi, A. Nafkha, Y. Nasser, O. Bazzi, and Y. Louët, "Simple and accurate closed-form approximation of the standard condition number distribution with application in spectrum sensing," CROWNCOM 2016, pp. 351-362, 2016.

[20] H. Kobeissi, Y. Nasser, A. Nafkha, O. Bazzi, and Y. Louët, "On the detection probability of the standard condition number detector in finite-dimensional cognitive radio context," EURASIP Journal on Wireless Communications and Networking, vol. 2016, no. 1, pp. 1-11, 2016. [Online]. Available: http://dx.doi.org/10.1186/s13638-016-0634-0
[21] L. Bixio, G. Oliveri, M. Ottonello, M. Raffetto, and C. S. Regazzoni, "Cognitive radios with multiple antennas exploiting spatial opportunities," IEEE Transactions on Signal Processing, vol. 58, no. 8, pp. 44534459, Aug 2010.

[22] H. Sarvanko, M. Höyhtyä, M. Matinmikko, and A. Mämmelä, "Exploiting spatial dimension in cognitive radios and networks," June 2011, pp. 360-364.

[23] H. Islam, Y. c. Liang, and A. T. Hoang, "Joint power control and beamforming for cognitive radio networks," IEEE Transactions on Wireless Communications, vol. 7, no. 7, pp. 2415-2419, July 2008.

[24] R. Zhang and Y. C. Liang, "Exploiting multi-antennas for opportunistic spectrum sharing in cognitive radio networks," IEEE Journal of Selected Topics in Signal Processing, vol. 2, no. 1, pp. 88-102, Feb 2008.

[25] R. Zhang, F. Gao, and Y. C. Liang, "Cognitive beamforming made practical: Effective interference channel and learning-throughput tradeoff," IEEE 10th Workshop on Signal Processing Advances in Wireless Communications, pp. 588-592, June 2009.

[26] T. Chen, H. Yuan, T. Zhao, Z. Zhang, and X. Ao, "Joint beamforming and power allocation for secure communication in cognitive radio networks," IET Communications, vol. 10, no. 10, pp. 1156-1162, 2016.

[27] N. Seifi, R. W. Heath, M. Coldrey, and T. Svensson, "Adaptive multicell 3-d beamforming in multiantenna cellular networks," IEEE Transactions on Vehicular Technology, vol. 65, no. 8, pp. 6217-6231, Aug 2016.

[28] E. G. Larsson, O. Edfors, F. Tufvesson, and T. L. Marzetta, "Massive mimo for next generation wireless systems," IEEE Communications Magazine, vol. 52, no. 2, pp. 186-195, February 2014.

[29] F. Boccardi, R. W. Heath, A. Lozano, T. L. Marzetta, and P. Popovski, "Five disruptive technology directions for 5g," IEEE Communications Magazine, vol. 52, no. 2, pp. 74-80, February 2014.

[30] W. H. Chin, Z. Fan, and R. Haines, "Emerging technologies and research challenges for $5 \mathrm{~g}$ wireless networks," IEEE Wireless Communications, vol. 21, no. 2, pp. 106-112, April 2014.

[31] J. Choi, V. Va, N. Gonzalez-Prelcic, R. Daniels, C. R. Bhat, and R. W. Heath, "Millimeter-wave vehicular communication to support massive automotive sensing," IEEE Communications Magazine, vol. 54, no. 12, pp. 160-167, December 2016.

[32] H. Sun, A. Nallanathan, C. X. Wang, and Y. Chen, "Wideband spectrum sensing for cognitive radio networks: a survey," IEEE Wireless Communications, vol. 20, no. 2, pp. 74-81, April 2013.

[33] K. Wanuga, N. Gulati, H. Saarnisaari, and K. R. Dandekar, "Online learning for spectrum sensing and reconfigurable antenna control," 9th International Conference on Cognitive Radio Oriented Wireless Networks and Communications (CROWNCOM), pp. 508-513, June 2014.

[34] Y. C. Liang, Y. Zeng, E. C. Y. Peh, and A. T. Hoang, "Sensingthroughput tradeoff for cognitive radio networks," IEEE Transactions on Wireless Communications, vol. 7, no. 4, pp. 1326-1337, April 2008.

[35] E. Ahmed, A. M. Eltawil, and A. Sabharwal, "Rate gain region and design tradeoffs for full-duplex wireless communications," IEEE Transactions on Wireless Communications, vol. 12, no. 7, pp. 3556-3565, July 2013.

[36] E. Björnson, M. Kountouris, and M. Debbah, "Massive mimo and small cells: Improving energy efficiency by optimal soft-cell coordination," May 2013, pp. 1-5.

[37] L. Wei, P. Dharmawansa, and O. Tirkkonen, "Locally best invariant test for multiple primary user spectrum sensing," Cognitive Radio Oriented Wireless Networks and Communications (CROWNCOM), 2012 7th International ICST article on, pp. 367-372, June 2012.

[38] M. Johnstone, "On the distribution of the largest eigenvalue in principal components analysis," Ann. Statist, vol. 29, pp. 295-327, 2001.

[39] C. G. Khatri, "Distribution of the largest or the smallest characteristic root under null hypothesis concerning complex multivariate normal populations," Ann. Math. Statist., vol. 35, no. 4, pp. 1807-1810, 12 1964. [Online]. Available: http://dx.doi.org/10.1214/aoms/1177700403

[40] A. Zanella, M. Chiani, and M. Win, "On the marginal distribution of the eigenvalues of wishart matrices," IEEE Trans. Commun., vol. 57, no. 4, pp. 1050-1060, April 2009.

[41] J. Baik and J. W. Silverstein, "Eigenvalues of large sample covariance matrices of spiked population models," Journal of Multivariate Analysis, vol. 97, no. 6, pp. 1382-1408, 2006.

[42] O. Tirkkonen and L. Wei, Foundation of Cognitive Radio Systems. InTech, 2012, ch. Exact and Asymptotic Analysis of Largest Eigenvalue Based Spectrum Sensing.

[43] J. Baik and J. W. Silverstein, "Eigenvalues of large sample covariance matrices of spiked population models," Journal of Multivariate Analysis, vol. 97, no. 6, pp. 1382-1408, 2006. 
[44] F. Bornemann, "On the numerical evaluation of distributions in random matrix theory: A review with an invitation to experimental mathematics," Markov Processes Relat. Fields, vol. 16, pp. 803-866, 2009.

[45] H. Kobeissi, A. Nafkha, Y. Nasser, Y. Louët, and O. Bazzi, "Approximating the standard condition number for cognitive radio spectrum sensing with finite number of sensors," IET Signal Processing, vol. 11, no. 2 , pp. 145-154, 2016.

[46] L. Wei and O. Tirkkonen, "Analysis of scaled largest eigenvalue based detection for spectrum sensing," Communications (ICC), IEEE International article on, pp. 1-5, June 2011.

[47] G. Marsaglia, "Ratios of normal variables," Journal of Statistical Software, vol. 16, no. 4, pp. 1-10, May 2006.

[48] H. Artes, D. Seethaler, and F. Hlawatsch, "Efficient detection algorithms for mimo channels: a geometrical approach to approximate $\mathrm{ml} \mathrm{detec-}$ tion," IEEE Trans. Signal Processing, vol. 51, no. 11, pp. 2808-2820,
Nov 2003

[49] J. Maurer, G. Matz, and D. Seethaler, "Low-complexity and full-diversity mimo detection based on condition number thresholding," IEEE Int. Conf. A.S.S.P., vol. 3, pp. 61-64, April 2007.

[50] V. Erceg, P. Soma, D. Baum, and A. J. Paulraj, "Capacity obtained from multiple-input multiple-output channel measurements in fixed wireless environments at $2.5 \mathrm{ghz}$, in Proc. IEEE Int. Conf. Commun. (ICC), vol. 1, pp. 396-400, May 2002.

[51] C. A. Tracy and H. Widom, "On orthogonal and symplectic matrix ensembles," Comm. Math. Phys., vol. 177, no. 3, pp. 727-754, 1996. [Online]. Available: http://projecteuclid.org/euclid.cmp/1104286442

[52] O. N. Feldheim and S. Sodin, "A universality result for the smallest eigenvalues of certain sample covariance matrices," Geometric and Functional Analysis, vol. 20, no. 1, pp. 88-123, 2010. [Online]. Available: http://dx.doi.org/10.1007/s00039-010-0055-x 